\title{
Investigation of the benefit of PVC in high resolution PET as a post and within reconstruction method for FDG brain data
}

Evangelos Raptis ( $\square$ raptisvag@gmail.com )

The University of Manchester https://orcid.org/0000-0003-2115-2639

\section{Laura Parkes}

The University of Manchester

Jose Anton-Rodriguez

The University of Manchester

\section{Stephen Carter}

The University of Manchester

\section{Karl Herholz}

The University of Manchester

Julian Matthews

The University of Manchester

Original research

Keywords: Positron Emission Tomography, Magnetic Resonance Imaging, Partial Volume Correction

Posted Date: July 22nd, 2020

DOl: https://doi.org/10.21203/rs.3.rs-44351/v1

License: (9) (i) This work is licensed under a Creative Commons Attribution 4.0 International License. Read Full License 


\title{
Investigation of the benefit of PVC in high resolution PET as a post and within reconstruction method for FDG brain data
}

\author{
Evangelos Raptis ${ }^{1}$, Laura M. Parkes ${ }^{2}$, Jose M. Anton-Rodriguez ${ }^{2}$, Stephen F. Carter ${ }^{2}$, Karl Herholz ${ }^{2}$ and \\ Julian C. Matthews ${ }^{2}$
}

\footnotetext{
${ }^{*}$ Correspondence: evange-

los.raptis@postgrad.manchester.ac.uk

${ }^{1}$ Department of Informatics,

Imaging and Data Sciences,

University of Manchester, WMIC,

Palatine road, Manchester, UK

Full list of author information is

available at the end of the article
}

\begin{abstract}
Purpose: The combination of positron emission tomography (PET) with magnetic resonance imaging (MRI) may enable novel research in the field of dementia. MR data is commonly used in the analysis of PET data for dementia due to its anatomical information and good soft tissue contrast. PET image reconstruction is currently performed independently of MRI data and the images typically suffer from low resolution, poor signal-to-noise ratio and count dependent bias, due to random error in acquired data and the reconstruction process which is ill conditioned. The aim of this research is to investigate the benefit of using anatomical information from MR data within PET image reconstruction, applied to dementia research.
\end{abstract}

Methods: Real PET and MRI patient data of 5 FDG scans of a healthy elderly volunteers, were used in order to create realistic ground truth images of the distribution of matter and activity for these individuals. These ground truth images underwent a Monte-Carlo simulation using SimSET, in order to generate simulated raw data of the high research resolution tomograph (HRRT) PET scanner. The simulations were validated by comparing the reconstructed images to real HRRT data and focusing on image resolution. A comparison of partial volume correction (PVC) of PET data applied within image reconstruction with the conventional approach of applying it post-reconstruction was conducted with typical count levels in order to evaluate the hypothesis that there would be benefit of applying PVC within image reconstruction.

Results: Results showed a little improvement in the recovered activity values is seen when using Lucy-Richardson deconvolution both post and within the image reconstruction. Similarly the use of RM modelling showed little benefit. Differences were observed when using Rousset PVC, with larger differences observed when interleaved with reconstruction. Generally the used of Rousset PVC within reconstruction resulted in a decrease in the bias (average error) for large cortical regions, but an increase in bias was observed for small regions and there were apparent region specific and patient specific variations in the observed bias.

Conclusions: The benefit of applying PVC as a reconstruction based method showed to be minimal. A region specific bias was observed for most of the reconstruction methods, either applied within or post image reconstruction. Further work is needed to evaluate the benefit of applying PVC methods for high resolution scanners.

Keywords: Positron Emission Tomography; Magnetic Resonance Imaging; Partial Volume Correction 


\section{Background}

Nuclear medicine and more specific PET imaging suffers from low spatial resolution, in addition to other deteriorating factors such as poor signal-to-noise and count dependent bias. Specifically, a PET image appears to be blurry compared to other imaging techniques such as $\mathrm{CT}$ and MRI. When trying to measure the radioactivity within a region this blurring results in a partial volume effect (PVE), where errors in the measurement occurs as a result of the spill-in and spill-out of radioactivity from one region to another. Firstly, the finite voxel size means that voxels can contain mixtures of tissues with different radioactivity distributions. Secondly the finite resolution of the images results in the mixture of radioactivity between image voxels with a loss of radioactivity due to the activity spilling out of a region of interest but also the contamination of the region through the spilling in of activity from adjacent structures. For PET the latter factor often dominates due to the low resolution in contrast to high resolution CT and MRI images where the former voxel size factor is dominant. Nevertheless both are contributing factors to the measurement of radioactivity with small structures.

In order to overcome this effect, a number of partial volume correction (PVC) methods have been proposed for PET images. These methods can be further divided into region-based and voxel-based approaches. The aim of these PVC techniques is to correct for the mislocation of radioactivity caused by the limited spatial resolution. In order to do this, in all cases, a model of the limited spatial resolution is needed. This is often modelled as the convolution of the PET image with a point spread function (PSF) kernel. Such an approach assumes that the blurring is spatially invariant, despite evidence that for most PET scanners the blurring typically become more extensive the further you get from the centre of the scanner FOV [1]. Volume-based methods often use anatomical information derived from a coregistered high resolution scan such as an MRI scan in order to segment the image volume. The High Resolution Research Tomograph (HRRT) (Siemens/CTI HRRT) is a very high resolution human brain only PET scanner and therefore will have less PVE compared to conventional PET-CT scanners. Nevertheless PVE will still be limiting for many applications in dementia research. Although the HRRT is now an old scanner, there is an increase in interest in higher resolution scanners such as the Biograph Vision PET/CT from Siemens ${ }^{\circledR}$ or the EXPLORER total body from United Imaging ${ }^{\circledR}$. Hence, the benefits of correction for PVE for higher resolution scanners is an important research question that needs to be further investigated.

\section{Application of PVC in dementia}

Due to the thin thickness of the brain cortex it is inevitable for PVE to occur when PET imaging is used. In the case of Alzheimer's disease or any other neurodegenerative disease where atrophy occurs in most of the cerebral tissue, the degree of the PVE is likely to be variable in the measured PET image. Consequently, there is the potential to misinterpret changes due to atrophy as changes in the uptake of radioactivity into small brain structures such as the cortex, with PVC potentially correcting for these misinterpretations of the image data. PVC methods can be divided into two categories: post-image reconstruction PVC which is what is typically done and the reconstruction based PVC which is less commonly used. 
PVC post image reconstruction

PVC using MR-based method, was shown to provide better results in data when the correlation between age and cortical CBF was examined. An increase in the PVE was observed to be greater in older patients, where atrophy is more predominant [2]. Moreover, atrophy correction of the PET data was performed using MR information for phantom and patient data [3]. FDG-PET images where corrected for PVE's using the GM volume segmented from MRI and convoluted with the resolution of the PET scanner (Mueller-Gaertner method). Results showed that the PET corrected image delivered more homogenous tracer distribution in the phantom while in the patient study, FDG activity in the bilateral perisylvian and medial frontal areas before PVC which was later resolved (after applying PVC) was observed. Similar outcome from an FDG-PET study, concluded that hypometabolism detected in the left orbitofrontal cortex and the right temporolimbic regions can be resolved using a modified Mueller-Gaertner method PVC [4]. This modified version of MuellerGaertner, uses a GTM correction in a set of regions which is between 3 and the number of voxels. Then the corrected value of $\mathrm{WM}$ is used as the mean value for the entire tissue of WM while Mueller-Gaertner is applied [5]. In Alzheimer's disease where atrophy occurs throughout the entire cortex, PVE's tend to be larger therefore deteriorating the quantitative accuracy of PET. An FDG-PET study in mild AD patients, examined the importance of PVC [6]. Application of PVC did not provide any benefit on the posterior cingulate gyri, the parietal and temporal lobes because the uptake was decreased while for the medial temporal lobe uptake maintained. Early-onset AD (EOAD) and late-onset AD (LOAD) patients were also used for the evaluation of FDG and PiB uptake in comparison to healthy controls [7]. A Videen's and Maeltzer's PVC method was implemented to a combined GM and WM region defined from a segmented $T_{1}$-weighted MRI scan. Similar patterns were observed for FDG and PiB on the voxel-wise analysis level, with and without the application of PVC. However in ROI analysis, a significant increase was detected in cortical regions for both PiB and FDG after the PVC.

In the recent years due to the development of fluorine-18 tracers, many amyloid PET studies have shifted towards imaging with these fluroinated tracers. A sufficient evaluation of many of the existing and recently proposed PVC methods was performed using simulated and real $\left[{ }^{18} F\right]$ flutemetamol PET data [8]. Data representing healthy subjects, MCI and AD patients were used to compare the modified Mueller-Gaertner (mMG) and the region based voxel-wise (RBV) PVC methods. A major increase in the SUVR on MCI and AD patients was observed when RBV correction was applied for the regions of frontal and parietal lobe in comparison to the uncorrected data. In the hippocampal region a difference in SUVR was observed from the uncorrected data when modified Mueller-Gartner PVC was used for both MCI and AD data. This bias from the uncorrected image could easily been explained by the PVE's that occur in the GM regions. The main drawback from this study was the lack of an amyloid ground truth image for AD and MCI patients, which could provide knowledge on the importance of PVC and show the respective bias from the ground truth when a PVC method was applied. Similar findings were observed in a recent study where the GTM method was applied on 5 people with $\mathrm{AD}$ and 6 healthy controls respectively using the amyloid tracers of $\left[{ }^{18} \mathrm{~F}\right]$ florbetapir 
and $\left[{ }^{11} C\right] \mathrm{PiB}$. Results showed that PVC increases the discrimination between $\mathrm{AD}$ patients and the healthy controls [9].

PVC within image reconstruction

Even though these before mentioned post reconstruction PVC methods provide improvements as a deconvolution application, however there is a sufficient amount of noise which they can not suppress. Hence, PVC within image reconstruction methods have been proposed for a better quantification of PET images. Taking into consideration the ideal scenario where the radiotracer distribution is the same for all of the voxels in the same soft tissue, the PVE of PET could bring a difference of distribution between PET and MRI. As a result, methods that include anatomical models from MR images within the image reconstruction in accordance with the functional distribution of the tracer in PET, could potentially provide a benefit.

Anatomical priors have been used within the image reconstruction, an approach which share similarities with PVC. In more detail, registered anatomical information were used, from PET/CT scanners, to govern the penalty term applied during every image update, combined with Fourier rebinning for 3D PET data image reconstruction [10]. Also, the use of Markov random field priors and joint information or entropy measures have been previously proposed [11]. Recently proposed PVC techniques use intensity signal to model the similarity between functional and anatomical images. This intensity prior generates uniformity in regions of the PET image, in which the equivalent MR image shows a similar homogeneity for the same regions [12]. An alternative approach was the use of PVC in sinogram space. An algorithm that estimates simultaneously, from the projection data, the activity concentration within a segmented tissue class inside a VOI has been developed and applied to SPECT [13]. Another method for anatomical incorporation into the PET reconstruction process based on kernel methods has also been proposed [14]. This kernel based anatomical method is derived from the MRI image feature which can be transferred into the PET data for simultaneous acquisition [15]. The use of the "Structural-Functional Synergistic" Resolution Recovery which has the ability to incorporate standardized anatomical information, was proposed for PVC in the brain PET [16]. This technique showed efficient recovery of functional information at high-resolution when MRI was used. An increase on the lesion-to-background ratio was observed when the local projection PVC method was applied while the authors concluded that they had better results when the PVC method was applied to images reconstructed with resolution modelling. Such resolution modelling image reconstruction that uses a measured response function within the reconstruction, has been shown to improve the spatial resolution [17, 18]. Other correction techniques for spill in effect were also compared for phantom and patient studies, where background correction within reconstruction among the methods of local projection and hybrid kernelized provided the best performance [19].

A limited number of studies exist on the application of PVC within image reconstruction, on HRRT data. Most of these studies include the modelling of the scanners' PSF with the reconstruction, so called resolution modelling image reconstruction, an option which is offered by the HRRT users community software, an approach that has been used and investigated in the past [17]. Results from the 
application of resolution modelling (PSF) reconstruction of PET images, suggests that neuroreceptor quantification is improved [20]. However, those results were not compared with any true distribution of the radioactivity. In another study the accuracy of a reconstruction-based PVC, which takes into account the PSF of the PET scanner was validated [21]. NEMA NU2 image quality phantom and 5 healthy volunteers using $\left[{ }^{11} C\right]$ flumazenil were scanned on a HR+ and and a HRRT scanner using the respective PSF of each of the scanners. It was concluded that the application of PSF as a PVC reconstruction based method for the HR+ agrees with the non-corrected for PVE HRRT data, providing evidence for the benefits of resolution modelling for the lower resolution HR+ scanner. Moreover one of the main concerns was whether the HRRT could compensate for partial volume effects in the presence of age-related brain atrophy and the aging effects of $5-H T_{2 A} R$ binding [22], using three PVC methods: the Meltzer, the Mueller-Gaertner and the Rousset method. Skepticism regarding the use of PVC on the HRRT for healthy subjects, because it might not be necessary in that the aging process is not uniform across the life span. This finding along with other studies $[23,24]$ updates the knowledge at that time on the importance and the benefit regarding the application of PVC on the HRRT data.

This research aims to expand this knowledge by including the comparison of the data corrected for PVE with the true distribution of the radioactivity (ground truth image).

\section{Methods}

Based on a previous work [25] the creation of 5 ground truth image was conducted for 5 different FDG healthy subjects in order to examine the variability between subjects, for investigations on the observed benefits and limitations.

\section{Ground truth images}

$\left[{ }^{18} F\right]$ fluorodeoxyglucose (FDG) PET scans of 5 healthy elderly volunteers, scanned at 40-60 min post-injection were used. The spatial model that was used to create those 5 FDG ground truth images was the same as before, only this time we restricted the metabolism of the tracer within the regions of GM and WM. The reason we did this assumption was because it is known that on an FDG-PET scan the tracer metabolises more on the GM than the WM (figure 1).

\section{HRRT simulations}

Data were simulated for the high resolution research tomograph (HRRT) for these ground truth images using SimSET [26]. The block detector configuration was selected [27] and the dimension of a two layer HRRT phoswich block was specified. For each ring, 72 blocks were arranged rotating the blocks to form the octagonal flat panel design of the HRRT. Thirteen rings were then combined to create the 936 block layout of an HRRT scanner (figure 2). Additionally attenuating matter representing the scanner tunnel and tungsten shielding (collimator) was included. As it was described in the previous chapter, the energy resolution of detection was set to $20 \%$ with a reference energy of $511 \mathrm{keV}$ as is typical for LSO/LYSO. The use of $20 \%$ as the energy resolution, is based on the desire to simulate a realistic HRRT 


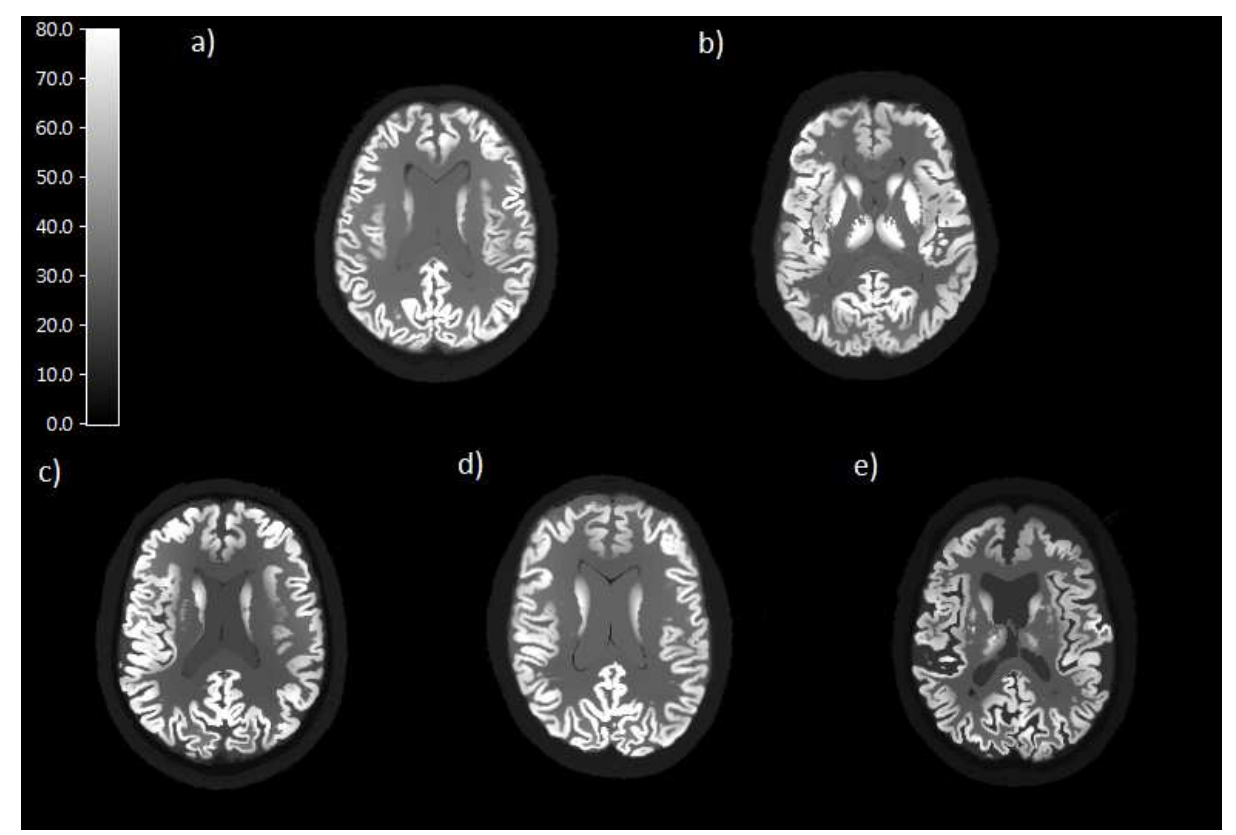

Figure 1 Axial presentation of the 5 FDG ground truth images (AU). Subjects shown a) h00013, b) h00016, c) h00021, d) h00029 and e) h00015. Metabolism between GM and WM was restricted while in CSF, bone and other soft tissue the intensity was constant. Image dimensions $256 \times 256 \times 150$, voxel size $=1 \mathrm{~mm}^{3}$

scanner. Knowing in fact that the typical energy resolution for a block is around $13 \%$, but in a complete calibration of the entire PET scanner, this resolution becomes even worst. Simulations were conducted using fluorine-18 for each of the test objects consisting of 25 million events every second (i.e. $25 \mathrm{MBq}$ of activity within the scanner field of view (FOV)) with simulations conducted lasting 1000 seconds in total.

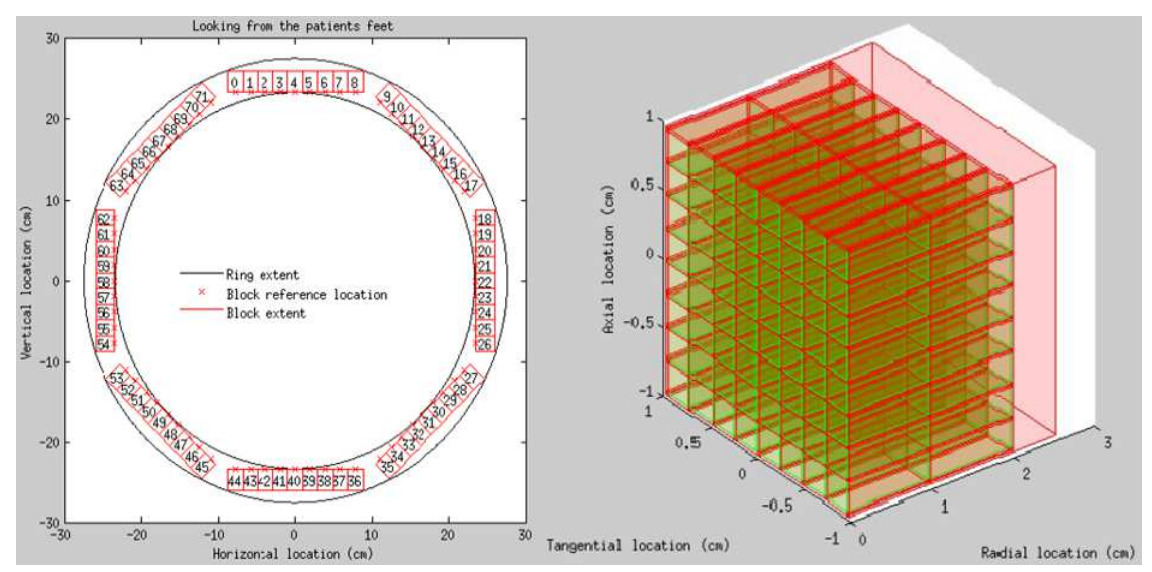

Figure 2 Structure of the tomograph (left) and three dimensional view of the location of materials within an HRRT block (Green = LSO/LYSO scintillation; Red = non-detection material) (right) 
Mapping of listmode files

The resulting simulated SimSET history files were converted into an HRRT listmode file using in house software. In addition, this conversion incorporated a random mispositioning of the events to adjacent crystals and different LSO/LYSO layers with the degree of this mispositioning adjusted [28]. Listmode files of trues, scatters and randoms were resampled into 5 different listmode events files and merged together in order to create 5 listmode files representing the prompt and delayed events, for each resampling [29]. The equivalent resampling values for FDG are described in table 1 . These data were subsequently reconstructed using the HRRT user community reconstruction software.

Table 1 Number of counts for the FDG patient's and simulated data along with the calculated sampling fractions

\begin{tabular}{|c|c|c|}
\hline FDG data & Events & Number of counts \\
\hline Patient & $n_{p}$ & 676 ' 916 ' 995 \\
counts & $n_{d}$ & $188^{\prime} 374^{\prime} 507$ \\
\hline Simulated & $N_{T}$ & 2'893'582'238 \\
data & $N_{S}$ & $1^{\prime} 763^{\prime} 153^{\prime} 229$ \\
counts & $N_{R}$ & $268^{\prime} 519^{\prime} 599$ \\
\hline Sampling & $f_{T}$ & 0.1049 \\
fractions & $f_{S}$ & 0.1049 \\
& $f_{R}$ & 0.7015 \\
\hline
\end{tabular}

\section{PVC implementations}

Two PVC methods were investigated:

1 Lucy-Richardson (LR) deconvolution: A Matlab code that performs LucyRichardson deconvolution was used [30]. This code was then compiled, in order to be used independently of Matlab and within image reconstruction as part of an iterative process of the HRRT reconstruction software.

2 Rousset: An implementation of the Rousset method [31] was developed, using Matlab. This code was cross-validated as showed in chapter 6 with the existing PETPVC toolbox [32]. This method requires regions which are assumed to be homogenous and which were created using the Hammers' atlas [33] and the $T_{1}$-weighted MR images using the regions that were predominantly grey matter, white matter, or cerebral spinal fluid. The recovered concentrations were then used to populate these regions creating a PVC corrected image.

Both methods require the point spread function (PSF) of the HRRT scanner to be defined. The default PSF kernel of the HRRT users community software was used which consist of a spatially invariant double isotropic Gaussian function (sum of two Gaussians with zero mean and FWHM (weight) values of: $2.12 \mathrm{~mm}$ and $5.89 \mathrm{~mm}$ ) [34]. An Analyze 7.5 or interfile formatted image file containing homogenous regions was created and used for Rousset PVC. The $T_{1}$-weighted MRI image that was used to create the ground truth was segmented into five different classes representing the grey matter (GM), white matter (WM), cerebrospinal fluid (CSF), bone and soft tissue. The Hammers' atlas which is composed by 83 regions in the brain was used in order to identify the existing regions within those classes and assign the number 
of voxels that exist in each region. Statistics to look at the number of voxels in each region for every single class was created and was written out in a csv file.

After identifying how many voxels of a region are in every class, the index image was created. The regions of CSF, bone, soft tissue and other, which is the subtraction of GM, WM, CSF, bone and soft tissue, were initially identified as unique and based on the $c s v$ file that was generated previously the remaining regions were determined as followed. The regions of Nucleus Accumbens, Pallidum, and Substancia Nigra for left and right were treated as a single region, meaning that a region which is only GM or WM. The regions of Hippocampus, Amygdala and Subcollsal area for left and right respectively were classified as a mixture of GM and CSF, while the Brainstem and the Corpus Collosum as a mixture of WM and CSF. All of the other regions were classified as a mixture between GM, WM, CSF and bone. The index images for the 5 FDG cases (figure 3), were then written out in Nifti format, with a voxel size similar to the PET image that will be used for PVC [25].

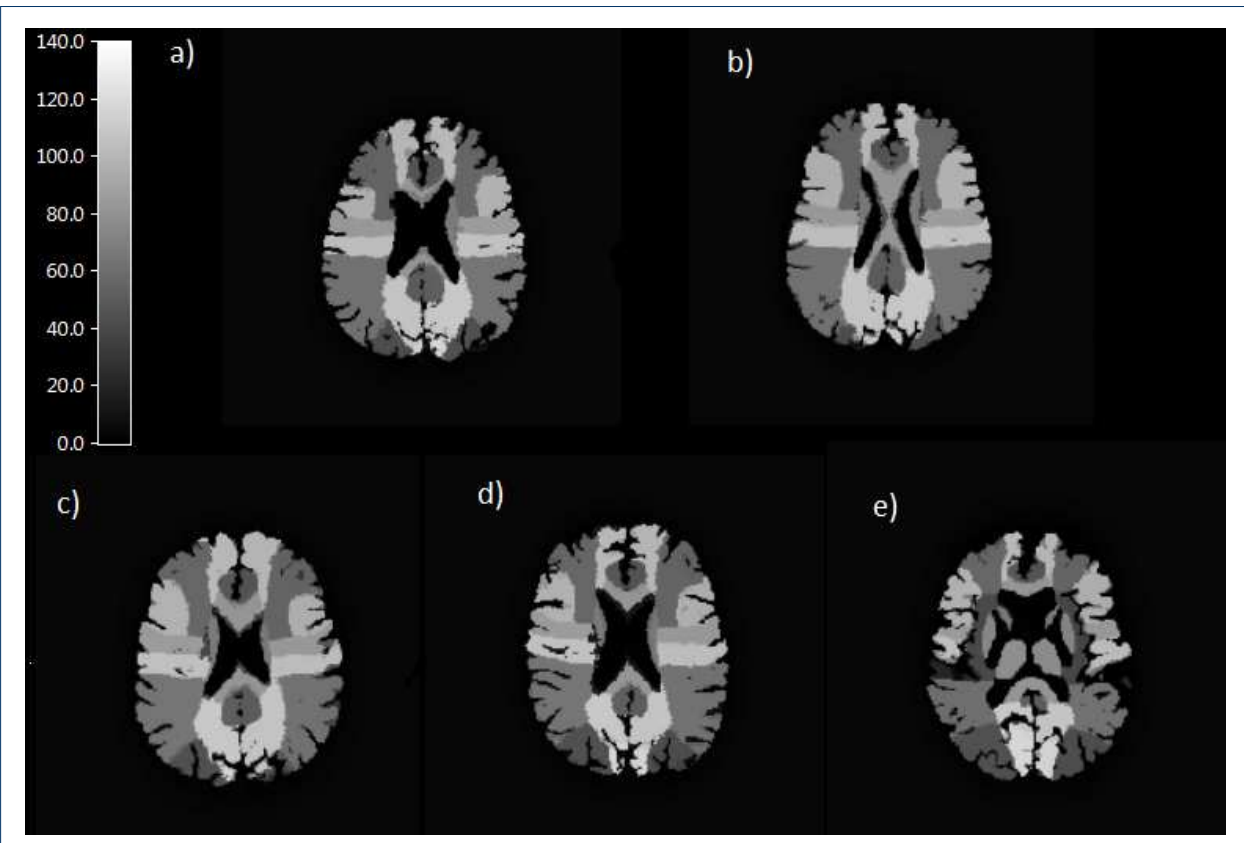

Figure 3 Axial view of FDG index images (in AU) for a) h00013, b) h00016, c) h00021, d) h00029 and e) h00015 created and used for Rousset PVC within and post image reconstruction

\section{PVC within image reconstruction}

PVC correction within image reconstruction was implemented by interleaving PVC of images with reconstruction iterations making use of previously developed theory [35]. In more detail, if we strip down the temporal component in this theory then assuming that the detection events follow a Poisson distribution for a random variable of emitted events $X_{j}$, where the mean number of emissions from the $j^{\text {th }}$ image voxel is modeled as $\lambda_{X_{j}}=f_{j}(\alpha)$, and which is described by any spatial model with parameter vector $\alpha$, the $\log$ likelihood problem is written as: 


$$
\begin{aligned}
& \alpha^{\text {opt }}=\underset{\alpha}{\arg \max } \sum_{i}\left(y_{i} \log _{e}\left(\lambda_{i}\right)-\lambda_{i}\right) \\
& \lambda_{i}=\eta_{i}+\sum_{j} p_{i, j} f_{j}(\alpha)
\end{aligned}
$$

where the $\log _{e}\left(y_{i} !\right)$ term is dropped as it is not dependent on $\alpha ; m_{i}$ is the number of detections in the $i^{\text {th }}$ projection bin; $\lambda_{i}$ is the predicted mean of $y_{i}$; and $\eta_{i}$ are the (known) mean number of erroneous events (scatter and randoms) in the $i^{\text {th }}$ projection bin. The system matrix $p_{i, j}$ is the probability of detection in the $i^{t h}$ bin given emission from the $j^{\text {th }}$ image voxel.

In order to solve it using the EM algorithm, where the complete data are the number of counts detected in the $i^{\text {th }}$ projection bin and which are emitted from the $j^{\text {th }}$ image voxel, then the E step is written as:

$$
E\left[\sum_{i j} \log _{e} P\left(m_{X_{j} ; Y_{i}} \mid \lambda\right) \mid y_{i}, \alpha^{(k)}\right]=\sum_{i j}\left(\hat{m}_{X_{j} ; Y_{i}} \log _{e}\left(p_{i, j} f_{j}(\alpha)\right)-p_{i, j} f_{j}(\alpha)\right.
$$

where $P\left(m_{X_{j} Y_{i}} \mid \alpha\right)$ is the probability of the complete data $m_{X_{j} Y_{i}}$ of a different random voxel variable $m$, given the model parameters; $\lambda^{(k)}$ is a current estimate of the parameters; and for the expectation of $\hat{m}$

$$
\hat{m}_{X_{j} Y_{i}}=E\left[m_{X_{j} Y_{i}} \mid y_{i}, \alpha^{(k)}\right]=\frac{p_{i, j} f_{j}\left(\alpha^{(k)}\right) y_{i}}{\eta_{i}+\sum_{j} p_{i, j} f_{j}\left(\alpha^{k}\right)}
$$

Rearranging equation (3) and maximising $\alpha$, the $\mathrm{M}$ step now becomes:

$$
\alpha^{(k+1)}=\underset{\alpha}{\arg \max } \sum_{j} s_{j}\left(\lambda_{X_{j}}^{(k+1)} \log _{e} f_{j}(\alpha)-f_{j}(\alpha)\right)
$$

where the sensitivity image is described by, $s_{j}=\sum_{i} p_{i, j}$ and the MLEM update for each frame now becomes:

$$
\lambda_{X_{j}}^{(k+1)}=\frac{f_{j}\left(\alpha^{(k)}\right)}{s_{j}} \sum_{i} \frac{p_{i, j} y_{i}}{\eta_{i}+\sum_{j} p_{i, j} f_{j}\left(\alpha^{k}\right)}
$$

The above equations were modified appropriately by removing the temporal component.

Specifically following each iteration a new partial volume corrected image was calculated, which was subsequently convolved with the PSF to create a blurred image which was used as the initial image estimate for the next iteration. The reconstruction iterations were conducted using the HRRT community implementation of the Ordered Subset Expectation Maximisation (OSEM) algorithm but with a single 
subset. LR deconvolution within image reconstruction using this approach is essentially the same as reconstruction using a resolution model (RM), nevertheless we also conducted reconstruction using the HRRT community reconstruction software with RM.

Image reconstruction

Various sets of reconstructed images were produced for all 5 listmode realisations. HRRT community software implementation of ordinary Poisson OSEM (OPOSEM) using 1 subset and 192 iterations, without any resolution modelling. OPOSEM HRRT user community software using 1 subset, 192 iterations and resolution modelling. Lucy-Richardson (LR) deconvolution with 1 iteration of OP-OSEM (1 subset) image reconstruction where every update from the previous iteration was blurred and used as the starting image for the next iteration with 192 interleaved iterations conducted. As above but using the compiled in-house software that performs Rousset PVC instead of LR deconvolution which was interleaved within the image reconstruction software. These reconstructions are summarised in (table 2). Additionally LR deconvolution and Rousset PVC was applied to the "Normal reconstruction" images, after the reconstruction (LR post, Rousset post). In total this resulted in 6 images for each realisation (with 5 realisations for the FDG simulation). The use of 192 iterations was principally chosen to match the number of updates (iterations $\times$ number of subsets) of reconstructions typically conducted using the HRRT scanner at the Wolfson Molecular Imaging Centre (WMIC) where 12 subsets with 16 iterations are used.

Table 2 Image reconstructions conducted using the HRRT users community software

\begin{tabular}{|c|c|c|c|c|c|}
\hline $\begin{array}{c}\text { Reconstruction } \\
\text { method }\end{array}$ & $\begin{array}{c}\text { Algorithm } \\
\text { used }\end{array}$ & Subsets & Iterations & PVC & $\begin{array}{c}\text { Resolution } \\
\text { modelling }\end{array}$ \\
\hline $\begin{array}{c}\text { Normal } \\
\text { reconstruction }\end{array}$ & OP-OSEM & 1 & 192 & No & Yes \\
\hline $\begin{array}{c}\text { RM } \\
\text { reconstruction }\end{array}$ & OP-OSEM & 1 & 192 & No & No \\
\hline LR within & OP-OSEM & 1 & 192 & $\begin{array}{c}\text { LR deconvolution } \\
\text { interleaved } \\
\text { with iterations }\end{array}$ & No \\
\hline Rousset within & OP-OSEM & 1 & 192 & $\begin{array}{c}\text { Rousset PVC } \\
\text { interleaved } \\
\text { with iterations }\end{array}$ & Nortions \\
\hline
\end{tabular}

Image analysis

Using the Hammers' atlas and the segmented $T_{1}$-weighted images, mean concentrations within concatenated GM regions were calculated for: the FDG simulated case and the 6 image datasets for each realisation. In addition these regions were applied to the simulated ground truth images enabling both the calculation of precision from the realisations; and error from comparison with the ground truth.

\section{Object map}

Based on the Hammers' atlas which was also used for the Rousset PVC, we created a Matlab code that produces an object map of the regions in the brain. This code 
selects the GM and the WM regions of the Hammers' atlas and calculates an image based on the sum of those two regions. A function then is applied and a 3D object map is produced. The Analyze 7.5 ${ }^{\circledR}$ software was utilised in order to visualise the reconstructed images for every PVC method and to check if the object map fits exactly on the reconstructed images and the ground truth image as well.

\section{Statistics}

After identifying this step, we produced the statistics file from the combination of the images with the object map. This file contained all the values needed in order to calculate the GM and WM values of each region (83 in total) in the brain. A weighted mean was calculated from the left and right part of each region in GM and WM using the following equation:

$$
\text { Weighted mean }=\frac{\left(\text { Mean }_{L} \times \text { Voxel }_{L}\right)+\left(\text { Mean }_{R} \times \text { Voxel }_{R}\right)}{\left(\text { Voxel }_{L}+\text { Voxel }_{R}\right)}
$$

A mean value from the 5 realisations of the GM and WM weighted mean (equation (7)), was calculated for all of the regions in the brain and for every reconstruction method.

$$
\text { Mean }=\sum_{1}^{n} \frac{\text { Weighted mean }}{n}, \text { where } n=\text { number of realisations }
$$

The standard deviation of this mean value based on the 5 realisations, was also computed to examine the precision of our results. Relative error was calculated by subtracting the mean value (GM mean value from the realisations) in every

reconstructed method from the respective value of the ground truth (GT) for the former and dividing with the value of the ground truth (equation (9)).

$$
\text { Relative error }=\frac{\text { Mean }-G T}{G T}
$$

\section{Results}

Results from the image reconstruction of the 5 FDG control subjects where we implemented the PVC methods of resolution modelling, Lucy-Richardson deconvolution and Rousset (within and post reconstruction for the two latter cases) is presented. Twenty (20) cortical regions were selected ordered from small to large in volume (from left to right). These regions are of high importance as they are valuable for assessing the accumulation of the tracer.

Image reconstructions for one of the FDG cases (h00015) along with the implementation of PVC are shown in figure 4. The remaining reconstructed images (h00013, h00016, h00021 and h00029) are presented in the additional file 1 section.

The relative error of the FDG reconstructed images (h00013, h00016, h00021, h00029 and h00015) are shown in figures 5, 6 and 7. 


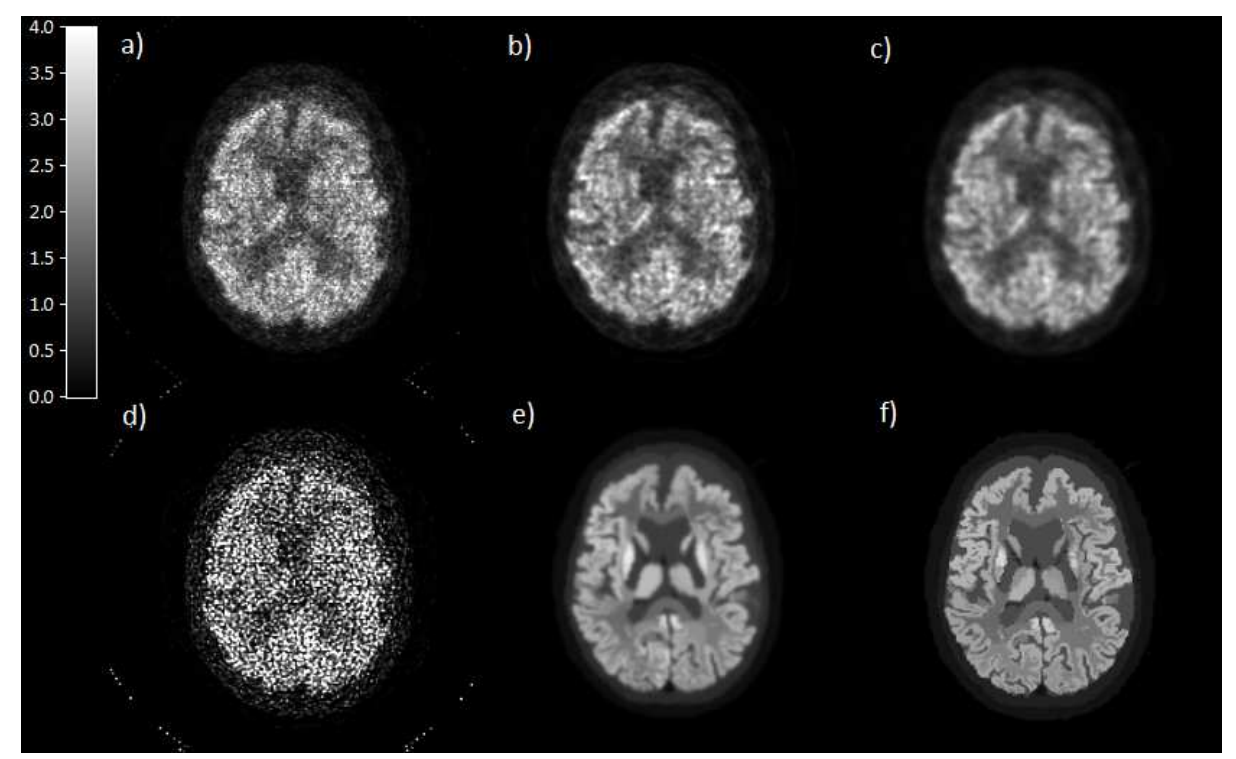

Figure 4 FDG h00015 image reconstruction (in AU) of the a) normal reconstruction, b) resolution modelling, c) Lucy-Richardson within reconstruction, d) Lucy-Richardson post reconstruction, e) Rousset within reconstruction, f) Rousset post reconstruction. Images were reconstructed using 3D OP-OSEM for 1 subset and 192 iterations. Image dimensions $256 \times 256 \times 207$, voxel size = $1.21875 \mathrm{~mm}^{3}$

The variation between the realisations remained small for all the FDG data, when observing the larger in size regions. Resolution modelling and LR deconvolution within image reconstruction provided a minimal or no benefit in the majority of the regions, but always had similar relative errors values. LR deconvolution as a post reconstruction PVC method, showed an increase in bias for 4/5 FDG data, performing even worst than the normal reconstruction. However, Rousset within image reconstruction showed a decrease in bias for the majority of the regions in h00016 and h00029, while an increase in bias was observed for the large regions in h00013, h00021 and h00015. A decrease in bias was also observed for Rousset post image reconstruction in the majority of the large areas in h00016 and h00029. For the cases of h00013, h00021 and h0015, Rousset post reconstruction PVC followed a similar pattern with the other methods (Normal reconstruction, RM and LR) without providing any benefit due to the small increase in bias for all the regions.

\section{Discussion}

The hypothesis of whether or not the application of PVC methods provide any benefit when applied within image reconstruction was evaluated. Prior to these results there was an expectation that PVC would reduce error but this would vary with the PVC method and whether this was done within image reconstruction or post reconstruction. PVC methods of Lucy-Richardson (LR) deconvolution and Rousset were applied both within and post reconstruction. The latter method requires regions of the brain to be homogenous which were created by the segmentation of a $T_{1}$-weighted MR image and the use of the Hammers' brain atlas. The calculation of the PSF of the scanner is also required for both of these methods as well as 

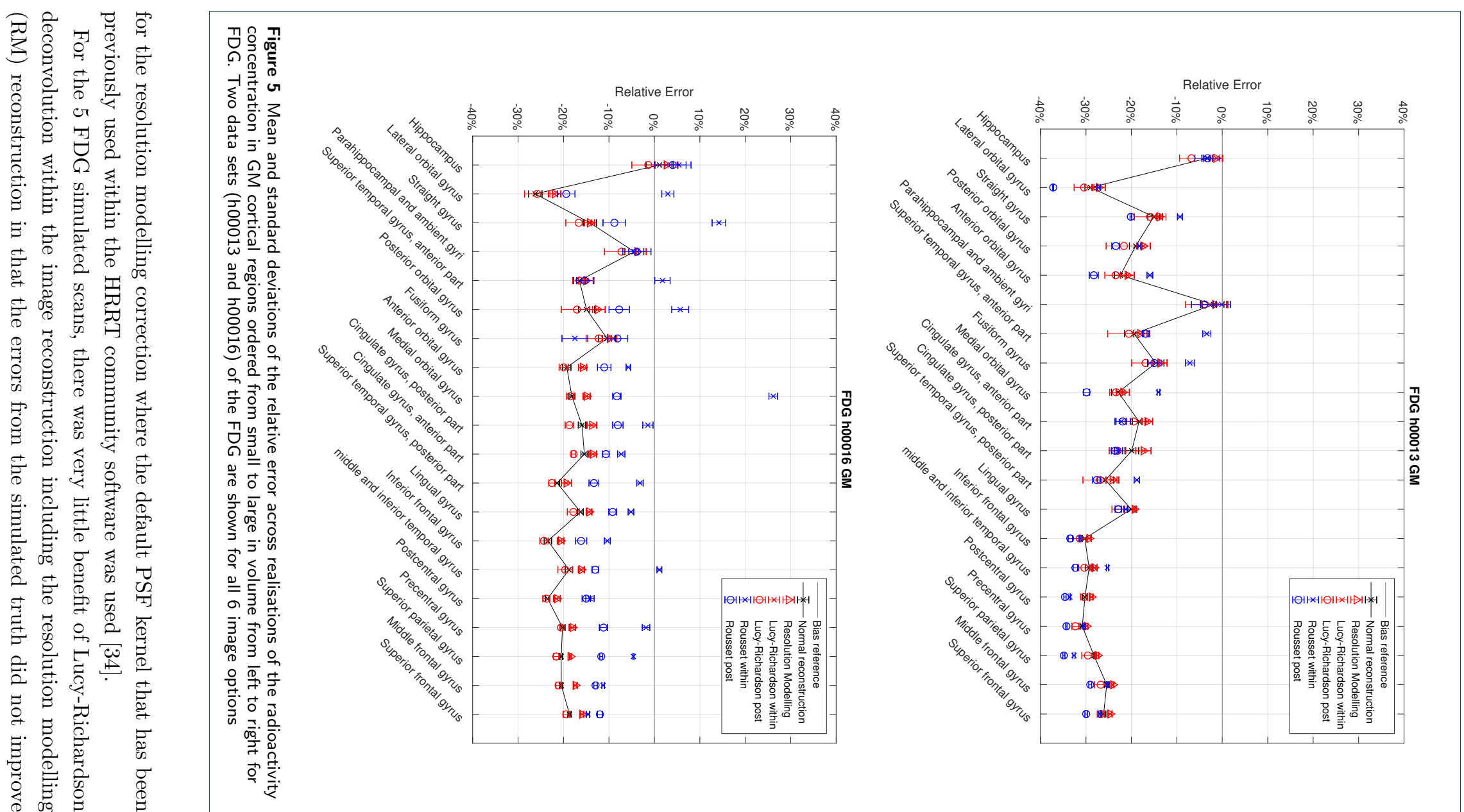

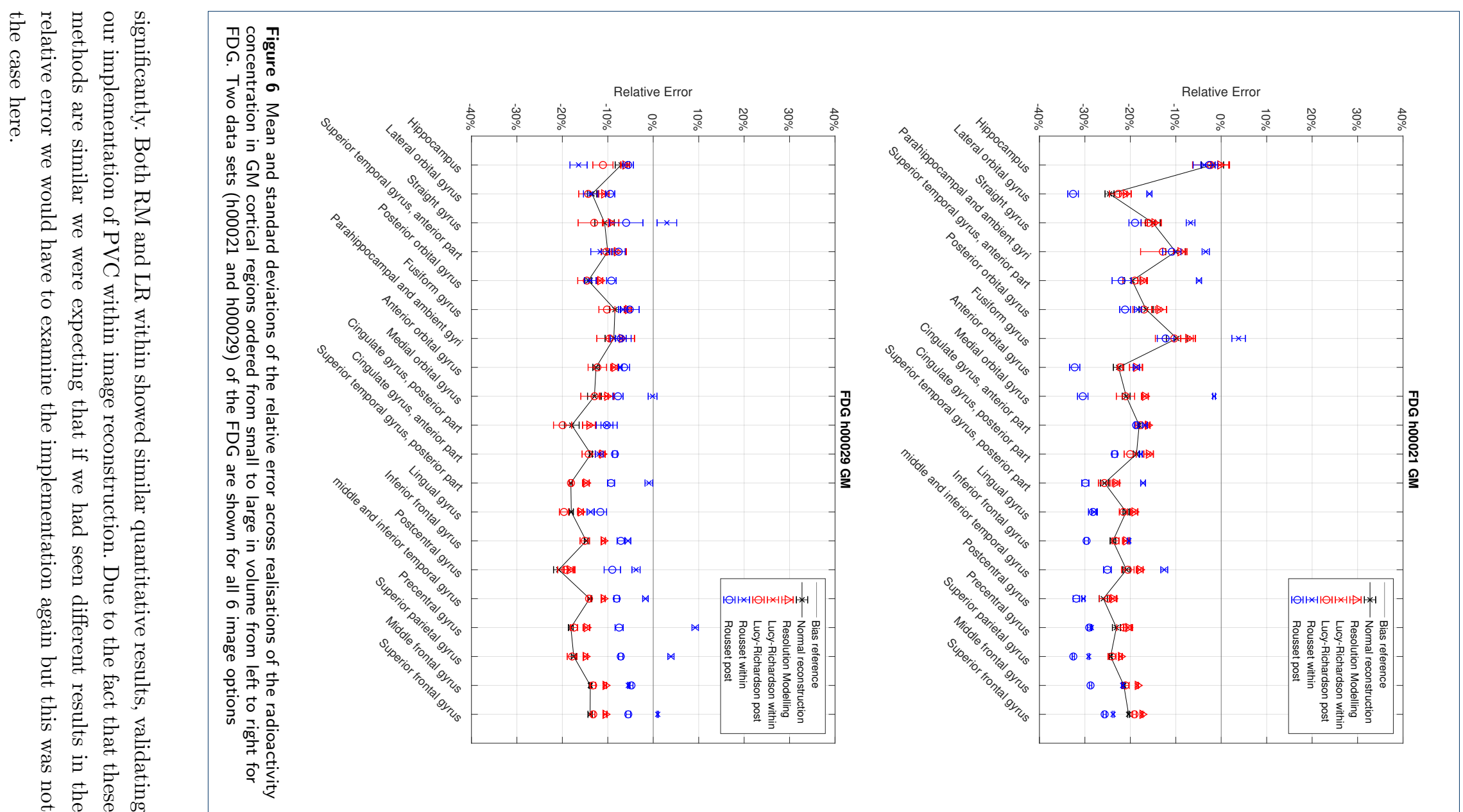


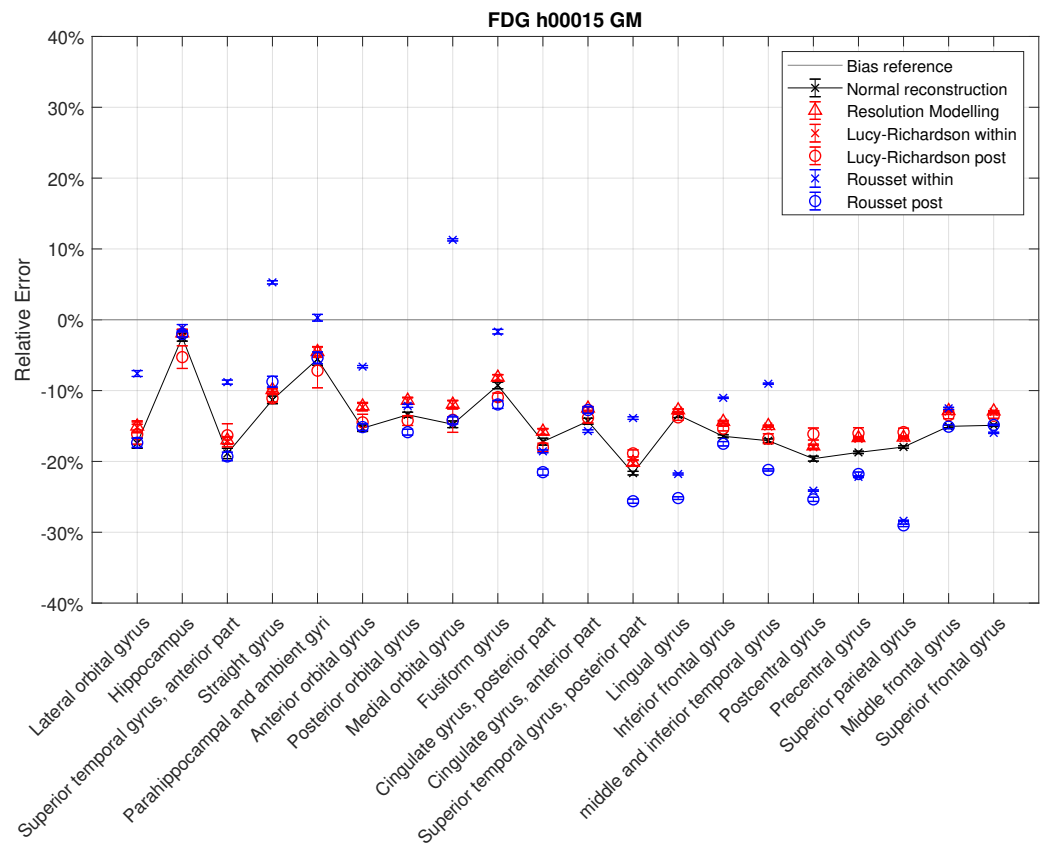

Figure 7 Mean and standard deviations of the relative error across realisations of the radioactivity concentration in GM cortical regions ordered from small to large in volume from left to right for FDG. Data from h00015 FDG are shown for all 6 image options

It can be observed from figures (5-7), the above methods showed a minimal reduction in bias (difference from the ground truth) in all 5 scans for all of the examined regions regardless their size, while the variance remained at low levels. This result agrees with the existing literature but also proves that there is only a small benefit regarding the application of RM and LR deconvolution for the HRRT scanner.

LR deconvolution as a post reconstruction method resulted in poor quality images with apparent high voxel variance for all the reconstructed listmode data of the 5 FDG scans. Variance remained at high levels in comparison with the rest of the reconstruction methods, in particular, variance was notably higher for the small regions. This result can be explained as deconvolution of the resulting image which is known to be ill-conditioned, resulting in an excessive noise. Additionally the kernel that was used for the LR deconvolution both within and post image reconstruction is more accurate in the centre of the FOV in comparison with the RM implementation of the HRRT's software, which is more conservative i.e. is narrower and therefore only partly recovers resolution.

Differences were observed when Rousset PVC was used either within or post image reconstruction. Large region specific variations in bias were observed in the FDG scans and which was apparently consistent between the simulations for the FDG scans. Rousset PVC within reconstruction showed a decrease of the relative error in 2/5 FDG cases for the majority of the cortical regions. For the rest 3 cases, PVC within reconstruction showed a decrease in bias mainly on the small in size regions. The exact explanation of this is not known but for instance this could be as a result of the homogeneity assumptions made with Rousset PVC. 
The use of Rousset PVC post image reconstruction showed a decrease on average in bias on the cortical regions, similar to that when Rousset was applied within image reconstruction for the 2 of the 5 (h00016 and h00029) FDG scans. Results from the large regions in the remaining FDG scans (h00013, h0021 and h00015), showed an increase in bias although this was less than Rousset PVC within reconstruction for the same areas. On the same FDG scans results, the application of Rousset PVC post image reconstruction showed a small decrease of the bias in the small cortical regions, although in most of the cases the benefit of Rousset post reconstruction was not apparent, because it was outperformed by the other methods such as normal reconstruction, resolution modelling and LR deconvolution. Precision remained at low levels between the realisations for the 5 FDG scans.

Overall the benefit of PVC was very modest with little differences observed between post and within reconstruction methods, where small benefits were observed when using Rousset PVC. The poor performance may reflect the already very high resolution of the HRRT with clearer benefits possible for a lower resolution scanner.

By comparing our results with some of the existing literature that can be relevant to our methodology we can identify similarities. For instance in [36], where quantitative evaluation of PET imaging using MRI based PVC methods was performed by comparing them with a FDG ground truth image. However in this study the scanner that was used was the HR+ which has a lower resolution to that of the HRRT (4.3 - $7.8 \mathrm{~mm}$ FWHM the former and $1.4-2.4 \mathrm{~mm}$ FWHM the latter). Also the ground truth reconstructed in this study was performed using a higher voxel size compared to our ground truth reconstruction. Moreover our research focuses more on the image based problem rather than the voxel based, hence the PVC methods that were selected and implemented differ. The use of anatomical prior in PET reconstruction was also evaluated [37]. However the simulations used in this study were not realistic due to inaccuracies of the tracer uptake and the entire study was focused on the thorax of the patient.

In a recent research [38] where the spill-in effect of counts was examined in amyloid negative subjects; the investigation of WM concentration was proved to be a valuable component in amyloid imaging. Using that same simulation software (SimSET) but for a different PET scanner, the acquisition times for the Monte-Carlo simulations was comparable to our methodology. The PETPVC toolbox which was cross-validated with our Rousset PVC method, was utilised for PVC and based on the results presented in this research for WM concentration, we can identify similarities with our results of WM distribution (Additional file 2). The deconvolution method and the region based method for PVC that was applied in this research show a similar behavior with the respective PVC methods that we used for the WM quantification, however it should be pointed that based on the examined subject there was a region specific bias and the values of the concentration without any PVC were not presented, in order to evaluate the benefit of applying PVC.

\section{Conclusion}

An evaluation of the benefit of PVC for the HRRT PET scanner was performed in FDG brain data. Based on previous work, FDG ground truth images were created using real PET and MRI data. These ground truth images underwent Monte-Carlo 
simulations for the HRRT scanner and the resulting data were reconstructed using the HRRT reconstruction software. Conventional reconstruction without PVC; resolution modelling reconstruction; Lucy-Richardson PVC post and within reconstruction; and Rousset PVC post and within reconstruction were implemented. Results showed a minimal benefit of applying PVC as a reconstruction based method. Region specific bias from the ground truth was observed for most of the reconstructed methods (-post or -within PVC) for both GM and WM. Further investigation would provide more information regarding the actual benefit of PVC for high resolution PET scanners.

\section{Acknowledgments}

The authors thank the people in the Wolfson Molecular Imaging Centre (WMIC) where the real PET and MRI data used for this study were initially acquired.

\section{Author's contributions}

All authors contributed in the elaboration of methods for data analysis, interpretation of results, experimental set-up and data collection, correction of manuscript, and approval. The simulation methodology and implementation of image reconstruction were mainly deployed by ER and JCM along with JMAR. The analysis of the results was proposed by SFC, applied by ER and evaluated by JCM, LMP and KH. Patient data were provided by SFC and KH. All authors read and approved the final manuscript.

\section{Funding}

This work is being conducted as part of a UK Engineering and Physical Sciences Research Council (EPSRC) funded Ph.D. with additional funding from the UK Medical Research Council Dementias Platform UK (DPUK) and the EPSRC Sensing and Imaging for Diagnosis of Dementias (SIDD) grant (EP/M005909/1).

Availability of data and materials

The data-sets used and/or analysed during the current study are available from the corresponding author on reasonable request.

Competing interests

The authors declare that they have no competing interests.

Ethics approval and consent to participate

Not applicable.

Consent for publication

Not applicable.

Author details

${ }^{1}$ Department of Informatics, Imaging and Data Sciences, University of Manchester, WMIC, Palatine road

Manchester, UK. ${ }^{2}$ Division of Neuroscience and Experimental Psychology, MAHSC, University of Manchester, Oxford road, Manchester, UK.

\section{References}

1. Kotasidis, F.A., Matthews, J.C., Angelis, G.I., Noonan, P.J., Jackson, A., Price, P., Lionheart, W.R., Reader, A.J.: Single scan parameterization of space-variant point spread functions in image space via a printed array: the impact for two PET/CT scanners. Physics in Medicine and Biology 56(10), 2917-2942 (2011)

2. Meltzer, C.C., Cantwell, M.N., Greer, P.J., Ben-Eliezer, D., Smith, G., Frank, G., Kaye, W.H., Houck, P.R., Price, J.C.: Does cerebral blood flow decline in healthy aging? A PET study with partial-volume correction. Journal of Nuclear Medicine 41(11), 1842-1848 (2000)

3. Yanase, D., Matsunari, I., Yajima, K., Chen, W.P., Fujikawa, A., Nishimura, S., Matsuda, H., Yamada, M.: Brain FDG PET study of normal aging in japanese: effect of atrophy correction. European Journal of Nuclear Medicine and Molecular Imaging 32(7), 794-805 (2005)

4. Curiati, P.K., Tamashiro-Duran, J.H., Duran, F.L.S., Buchpiguel, C.A., Squarzoni, P., Romano, D.C., Vallada, H., Menezes, P.R., Scazufca, M., Busatto, G.F., Alves, T.: Age-related metabolic profiles in cognitively healthy elders: Results from a voxel-based F-18 fluorodeoxyglucose positron emission tomography study with partial volume effects correction. American Journal of Neuroradiology 32(3), 560-565 (2011)

5. Quarantelli, M., Berkouk, K., Prinster, A., Landeau, B., Svarer, C., Balkay, L., Alfano, B., Brunetti, A., Baron, J.C., Salvatore, M.: Integrated software for the analysis of brain PET/SPECT studies with partial-volume-effect correction. Journal of Nuclear Medicine 45(2), 192-201 (2004)

6. Samuraki, M., Matsunari, I., Chen, W.P., Yajima, K., Yanase, D., Fujikawa, A., Takeda, N., Nishimura, S., Matsuda, H., Yamada, M.: Partial volume effect-corrected FDG PET and grey matter volume loss in patients with mild Alzheimer's disease. European Journal of Nuclear Medicine and Molecular Imaging 34(10), 1658-1669 (2007)

7. Rabinovici, G.D., Furst, A.J., Alkalay, A., Racine, C.A., O'Neil, J.P., Janabi, M., Baker, S.L., Agarwal, N., Bonasera, S.J., Mormino, E.C., Weiner, M.W., Gorno-Tempini, M.L., Rosen, H.J., Miller, B.L., Jagust, W.J.: Increased metabolic vulnerability in early-onset Alzheimer's disease is not related to amyloid burden. Brain (2010) 
8. Thomas, B.A., Erlandsson, K., Modat, M., Thurfjell, L., Vandenberghe, R., Ourselin, S., Hutton, B.F.: The importance of appropriate partial volume correction for PET quantification in Alzheimer's disease. European Journal of Nuclear Medicine and Molecular Imaging 38(6), 1104-1119 (2011)

9. Wong, D., Rousset, O., Nandi, A., Pontecorvo, M., Kitzmiller, K., Rosenberg, P., Roberts, J., George, N., Brasic, J., Marashdeh, W., Gapasin, L., Schmidt, M.: Role of partial volume correction in paired studies of C-11 PiB vs F-18 florbetapir: Implications for centiloid? Journal of Nuclear Medicine 59 (2018)

10. Alessio, A.M., Kinahan, P.E.: Improved quantitation for PET/CT image reconstruction with system modeling and anatomical priors. Medical Physics 33(11), 4095-4103 (2006)

11. Bai, B., Li, Q., Leahy, R.M.: Magnetic resonance-guided positron emission tomography image reconstruction Seminars in Nuclear Medicine 43(1), 30-44 (2013)

12. Tang, J., Rahmim, A.: Bayesian PET image reconstruction incorporating anato-functional joint entropy. Physics in Medicine and Biology 54(23), 7063-7075 (2009). 29

13. Moore, S.C., Southekal, S., Park, M.A., McQuaid, S.J., Kijewski, M.F., Muller, S.P.: Improved regional activity quantitation in nuclear medicine using a new approach to correct for tissue partial volume and spill-over effects. IEEE Transactions on Medical Imaging 31(2), 405-416 (2012)

14. Wang, G., Qi, J.: PET image reconstruction using kernel method. IEEE Transactions in Medical Imaging 34 61-71 (2015)

15. Chen, K.T., Salcedo, S., Gong, K., Chonde, D.B., Izquierdo-Garcia, D., Drzezga, A.E.: An efficient approach to perform MR-assisted PET data optimization in simultaneous PET/MR neuroimaging studies. Journal of Nuclear Medicine 60, 272-278 (2019)

16. Shidahara, M., Tsoumpas, C., Hammers, A., Boussion, N., Visvikis, D., Ito, H., Kimura, Y., Suhara, T., Kanno, I., Turkheimer, F.: Functional and structural synergy for resolution recovery and partial volume correction in brain PET. Journal of Cerebral Blood Flow and Metabolism 29, 73-74 (2009)

17. Panin, V.Y., Kehren, F., Michel, C., Casey, M.: Fully 3-D PET reconstruction with system matrix derived from point source measurements. IEEE Transactions on Medical Imaging 25(7), 907-921 (2006)

18. Rahmim, A., Qi, J.Y., Sossi, V.: Resolution modeling in PET imaging: Theory, practice, benefits, and pitfalls. Medical Physics 40(6) (2013)

19. Akerele, M. I. and Karakatsanis, N. A. and Deidda, D. and Cal-Gonzalez, J. and Forsythe, R. O. and Dweck, M.R. and Syed, M. and Newby, D. E. and Aykroyd. R. G. and Sourbron, S. and Tsoumpas, C.: Comparison of Correction Techniques for the Spill in Effect in Emission Tomography. IEEE Transactions on Radiation and Plasma Medical Sciences (2020)

20. Varrone, A., Sjoholm, N., Eriksson, L., Gulyas, B., Halldin, C., Farde, L.: Advancement in PET quantification using 3D-OP-OSEM point spread function reconstruction with the HRRT. European Journal of Nuclear Medicine and Molecular Imaging 36(10), 1639-1650 (2009)

21. Mourik, J.E.M., Lubberink, M., van Velden, F.H.P., Kloet, R.W., van Berckel, B.N.M., Lammertsma, A.A., Boellaard, R.: In vivo validation of reconstruction-based resolution recovery for human brain studies. Journal of Cerebral Blood Flow and Metabolism 30(2), 381-389 (2010)

22. Uchida, H., Chow, T.W., Mamo, D.C., Kapur, S., Mulsant, B.H., Houle, S., Pollock, B.G., Graff-Guerrero, A.: Effects of aging on 5-HT2AR binding: A HRRT PET study with and without partial volume corrections. International Journal of Geriatric Psychiatry 26(12), 1300-1308 (2011)

23. Leroy, C., Comtat, C., Trebossen, R., Syrota, A., Martinot, J.L., Ribeiro, M.J.: Assessment of (11)C-PE2I binding to the neuronal dopamine transporter in humans with the high-spatial-resolution PET scanner HRRT. Journal of Nuclear Medicine 48(4), 538-546 (2007)

24. Van Velden, F.H.P., Kloet, R.W., van Berckel, B.N.M., Buijs, F.L., Luurtsema, G., Lammertsma, A.A. Boellaard, R.: HRRT Versus HR plus Human Brain PET Studies: An Interscanner Test-Retest Study. Journal of Nuclear Medicine 50(5), 693-702 (2009)

25. Raptis, E. and Parkes, L. M. and Anton-Rodriguez, J. M. and Carter, S. F. and Herholz, K. and Matthews, J. C.: Evaluation of the Benefit of Partial Volume Correction for High Resolution PET Scanners. In: 2019 IEEE Nuclear Science Symposium and Medical Imaging Conference (NSS/MIC), pp. 1-3 (2019)

26. Division of Nuclear Medicine, University of Wahington: Simulation System for Emission Tomography (SimSET) (1993)

27. Harrison, R.L., Gillispie, S.B., Lewellen, T.K.: Design and implementation of a block detector simulation in SimSET. In: Nuclear Science Symposium Conference Record, 2006. IEEE, vol. 5, pp. 3151-3153 (2006). IEEE

28. Raptis, E., Parkes, L.M., Anton-Rodriguez, J., Carter, S.F., Herholz, K., Matthews, J.C., leee: Validation of a realistic simulation of the HRRT using SimSET. In: IEEE Nuclear Science Symposium and Medical Imaging Conference (NSS/MIC) (2017)

29. Markiewicz, P., Reader, A., Matthews, J.: Assessment of bootstrap resampling performance for PET data. Physics in medicine and biology 60(1), 279 (2014)

30. Segobin, S.H., Matthews, J.C., Markiewicz, P.J., Herholz, K., leee: A hybrid Between Region-Based and Voxel-Based Methods for Partial Volume Correction in PET. In: IEEE Nuclear Science Symposium (NSS)/Medical Imaging Conference (MIC)/17th International Workshop on Room-Temperature Semiconductor X-ray and Gamma-ray Detectors, pp. 3073-3078 (2010)

31. Rousset, O.G., Ma, Y.L., Evans, A.C.: Correction for partial volume effects in PET: Principle and validation. Journal of Nuclear Medicine 39(5), 904-911 (1998)

32. Thomas, B.A., Cuplov, V., Bousse, A., Mendes, A., Thielemans, K., Hutton, B.F., Erlandsson, K.: PETPVC: A toolbox for performing partial volume correction techniques in positron emission tomography. Physics in Medicine and Biology 61(22), 7975-7993 (2016)

33. Hammers, A., Allom, R., Koepp, M.J., Free, S.L., Myers, R., Lemieux, L., Mitchell, T.N., Brooks, D.J., Duncan, J.S.: Three-dimensional maximum probability atlas of the human brain, with particular reference to the temporal lobe. Human Brain Mapping 19(4), 224-247 (2003)

34. Comtat, C., Sureau, F.C., Sibomana, M., Hong, I.K., Sjoholm, N., Trebossen, R., leee: Image based resolution modeling for the HRRT OSEM reconstructions software. In: IEEE Nuclear Science Symposium/Medical 
Imaging Conference, pp. 3395-3398 (2009)

35. Matthews, J.C., Angelis, G.I., Kotasidis, F.A., Markiewicz, P.J., Reader, A.J., leee: Direct Reconstruction of Parametric Images Using Any Spatiotemporal 4D Image Based Model and Maximum Likelihood Expectation Maximisation. In: IEEE Nuclear Science Symposium (NSS)/Medical Imaging Conference (MIC)/17th International Workshop on Room-Temperature Semiconductor X-ray and Gamma-ray Detectors, pp. 2435-2441 (2010)

36. Vunckx, K., Atre, A., Baete, K., Reilhac, A., Deroose, C.M., Van Laere, K., Nuyts, J.: Evaluation of Three MRI-Based Anatomical Priors for Quantitative PET Brain Imaging. IEEE Transactions on Medical Imaging 31(3), 599-612 (2012)

37. Tsai, Y.J., Schramm, G., Ahn, S., Bousse, A., Arridge, S., Nuyts, J., Hutton, B.F., Stearns, C.W., Thielemans, K.: Benefits of Using a Spatially-Variant Penalty Strength With Anatomical Priors in PET Reconstruction. IEEE Transactions on Medical Imaging 39(1), 11-22 (2020)

38. Lopez-Gonzalez, F.J., Moscoso, A., Efthimiou, N., Fernandez-Ferreiro, A., Pineiro-Fiel, M., Archibald, S.J., Aguiar, P., Silva-Rodriguez, J.: Spill-in counts in the quantification of F-18-florbetapir on A-beta negative subjects: the effect of including white matter in the reference region. EJNMMI Physics 6(1) (2019)

\section{Additional Files}

Additional file 1 - Remaining FDG reconstructed images

The remaining FDG ground truth reconstructed images that were not included in the main text are presented here (figure 8). The data were reconstructed using normal reconstruction, resolution modelling, Lucy-Richardson (within and post) and Rousset (within and post) which were appropriately implemented on the HRRT users community reconstruction software.

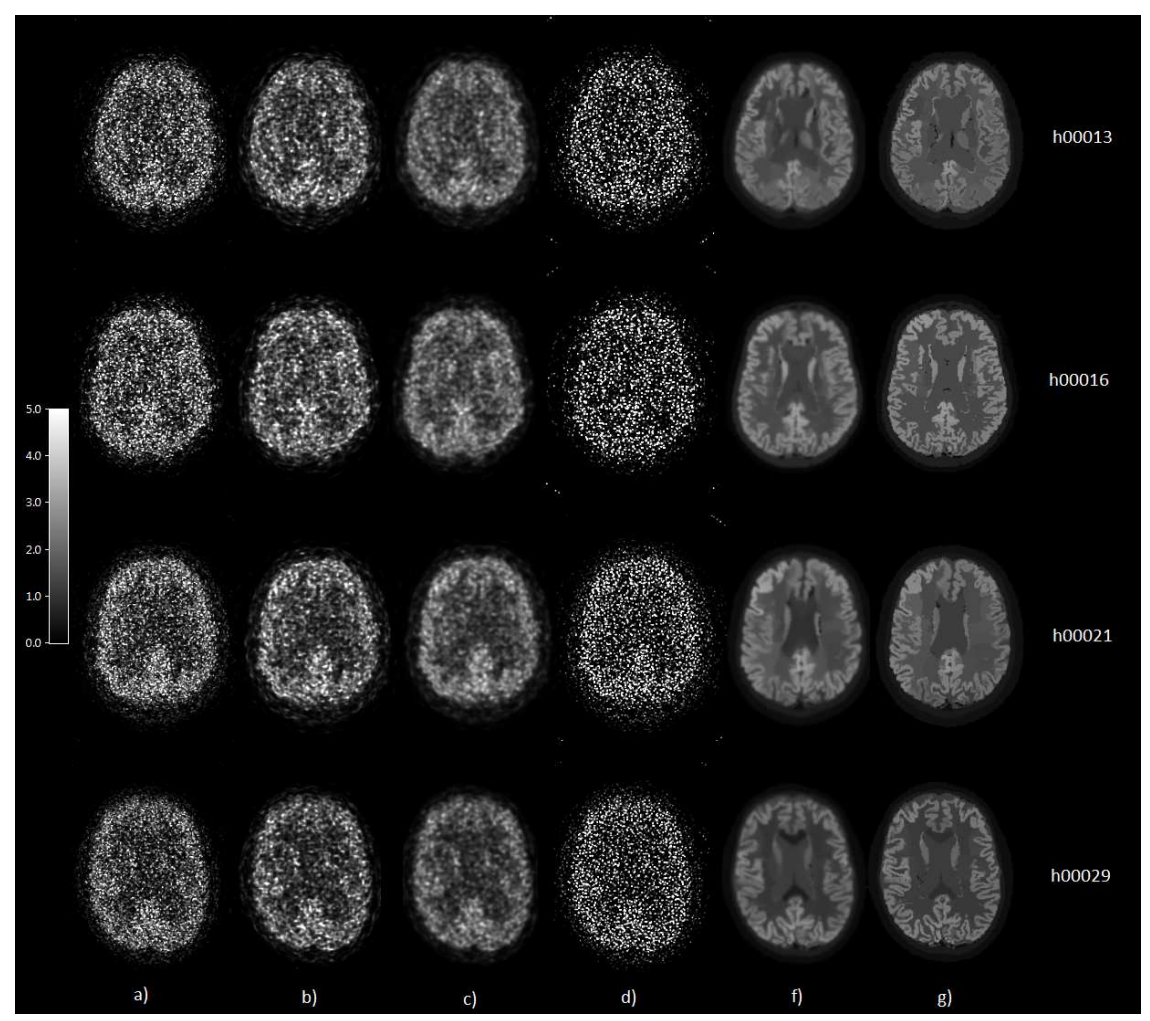

Figure 8 Remaining FDG reconstructed images (h00013, h00016, h00021 and h00029 in AU) of the a) normal reconstruction, b) resolution modelling, c) Lucy-Richardson within reconstruction, d) Lucy-Richardson post reconstruction, e) Rousset within reconstruction, f) Rousset post reconstruction. Images were reconstructed using 3D OP-OSEM for 1 subset and 192 iterations. Image dimensions $256 \times 256 \times 207$, voxel size $=1.21875 \mathrm{~mm}^{3}$ 
Additional file 2 - WM plots of relative bias for FDG

The distribution of the relative error for WM was also plotted for all of our results (figures $9,10,11$ ). In these figures we can observe that there is a reduction of the relative bias in WM when Rousset within was used, but due to the fact that we are interested mainly on the GM distribution we did not take these WM results as a major component of this research, even though the PVC methods seem to provide a benefit.
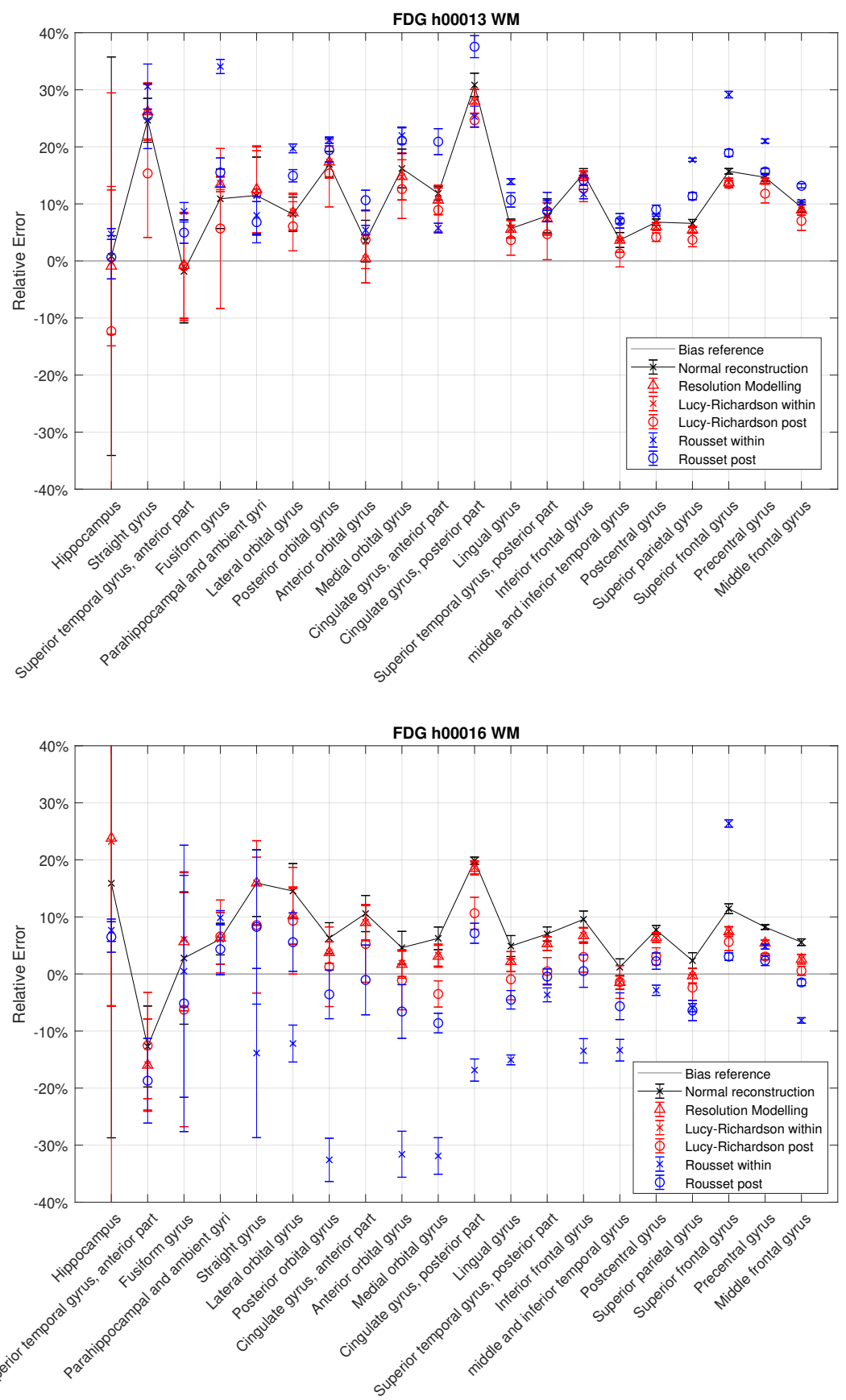

Figure 9 Mean and standard deviations of the relative error across realisations of the radioactivity concentration in WM cortical regions ordered from small to large in volume from left to right for FDG. Two data sets (h00013 and h00016) of the FDG are shown for all 6 image options 


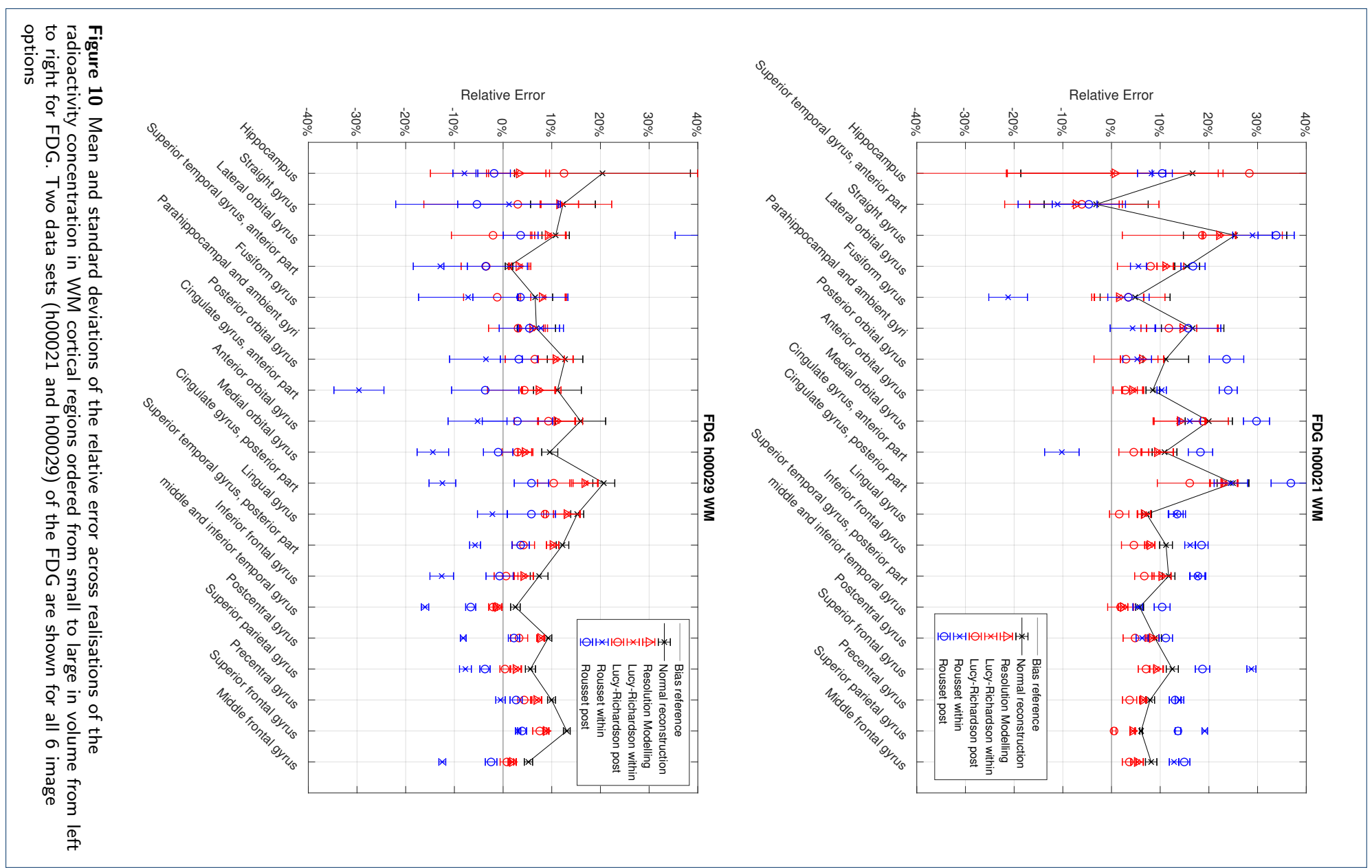




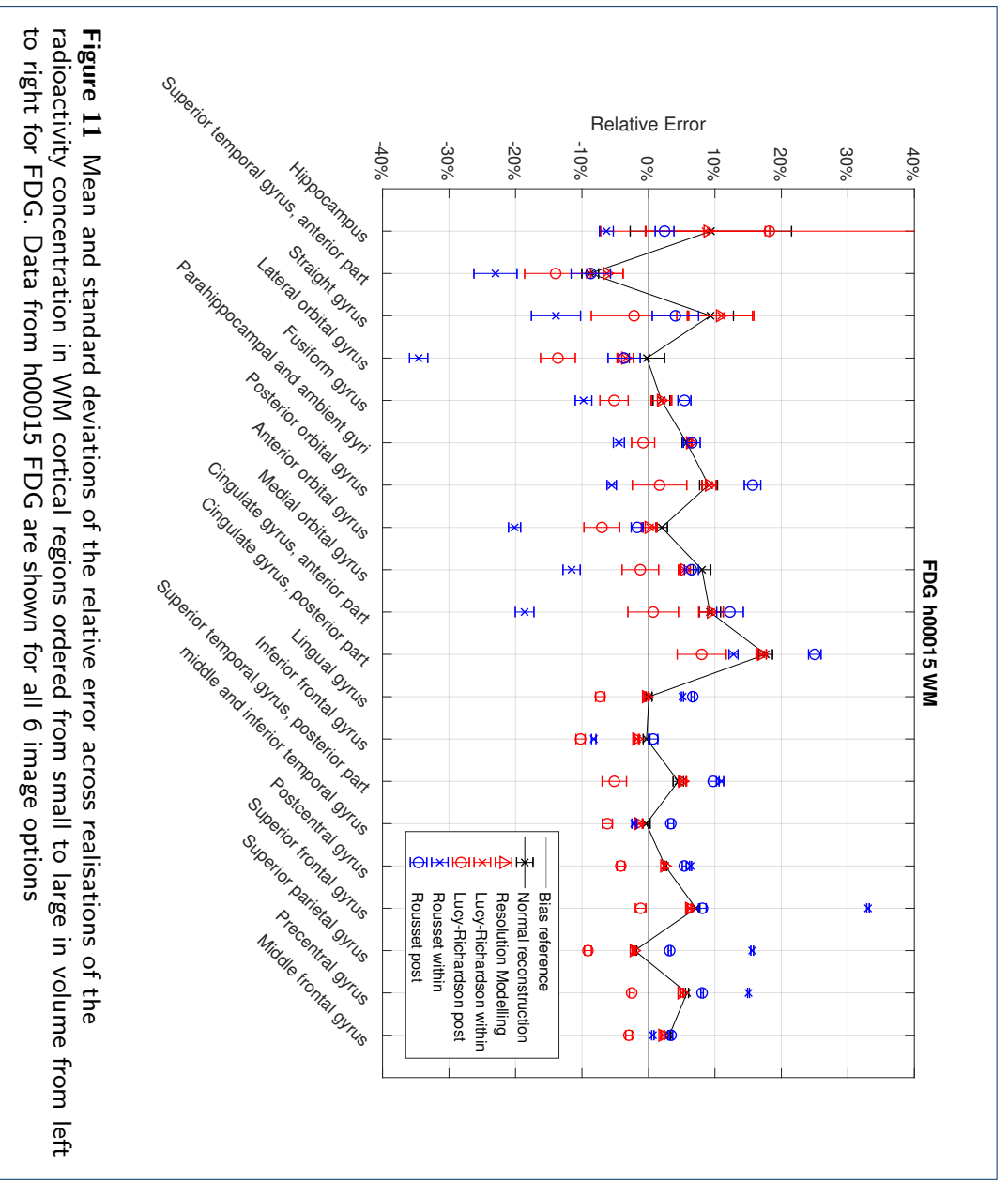




\section{Figures}

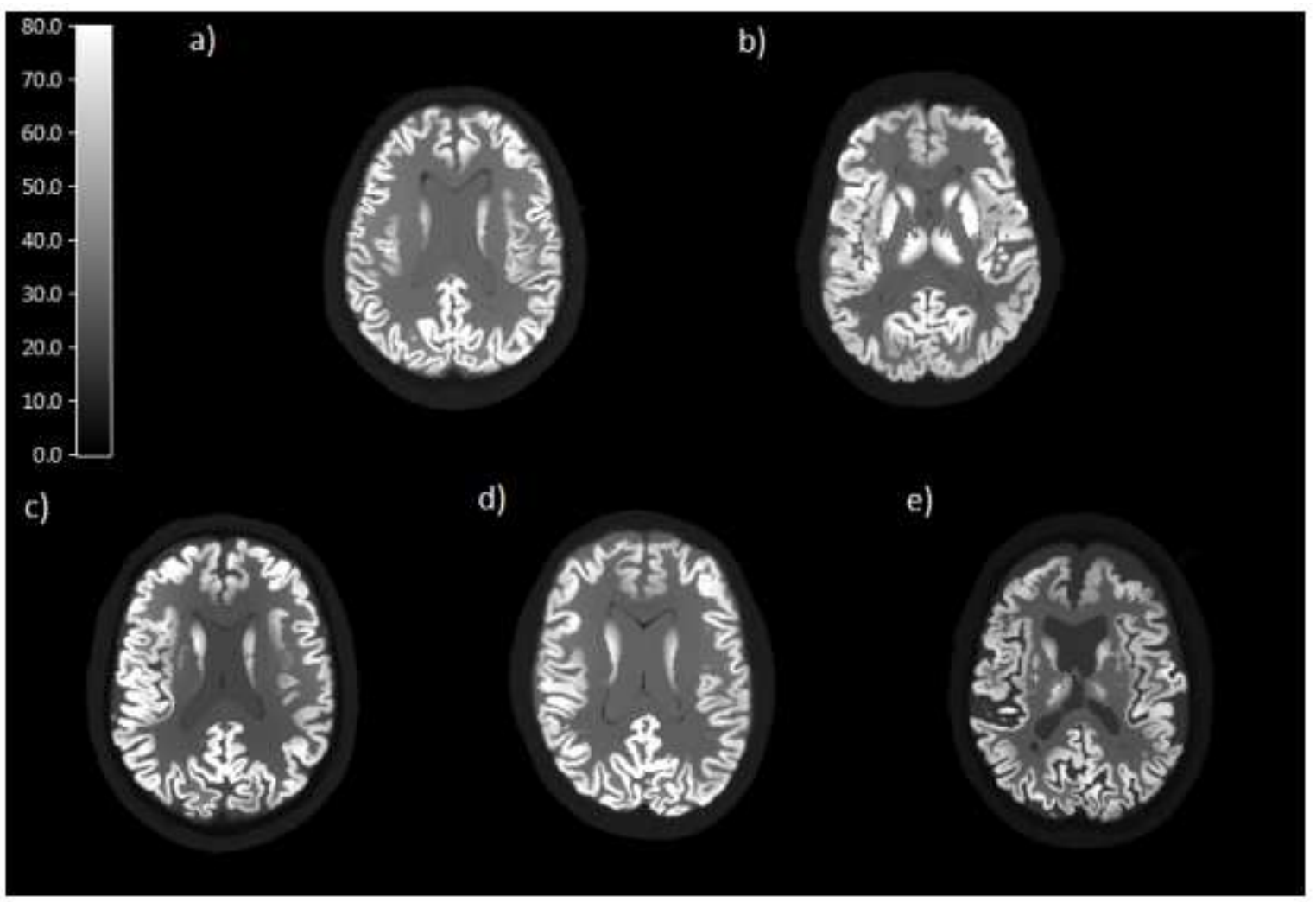

Figure 1

Axial presentation of the 5 FDG ground truth images (AU). Subjects shown a) h00013, b) h00016, c) h00021, d) h00029 and e) h00015. Metabolism between GM and WM was restricted while in CSF, bone and other soft tissue the intensity was constant. Image dimensions $256 \times 256 \times 150$, voxel size $=1 \mathrm{~mm} 3$

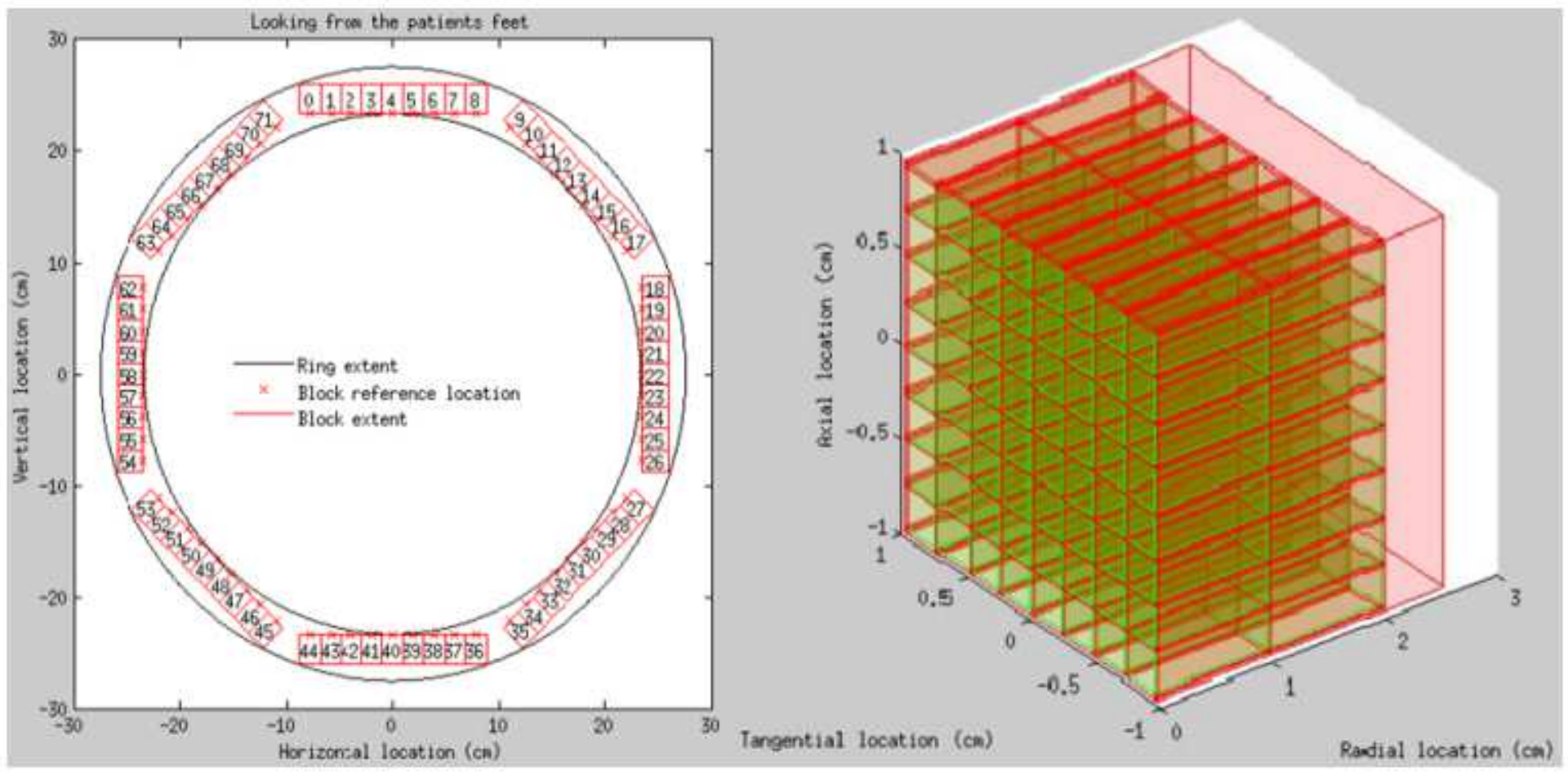




\section{Figure 2}

Structure of the tomograph (left) and three dimensional view of the location of materials within an HRRT block (Green = LSO/LYSO scintillation; Red = non-detection material) (right)

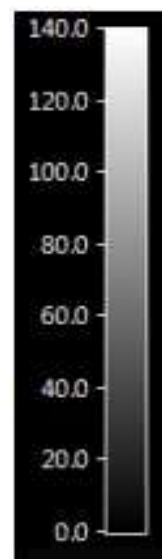

c)

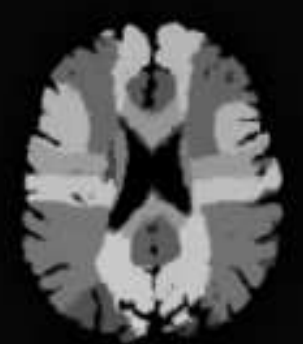

a)

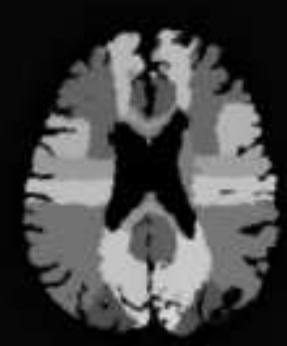

b)

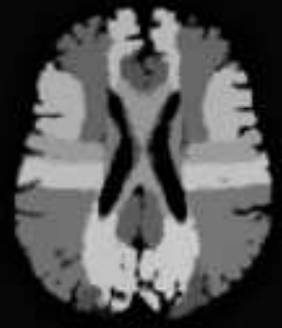

d)

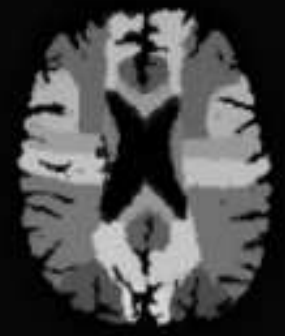

e)

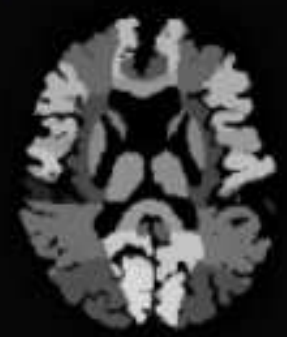

\section{Figure 3}

Axial view of FDG index images (in AU) for a) h00013, b) h00016, c) h00021, d) h00029 and e) h00015 created and used for Rousset PVC within and post image reconstruction

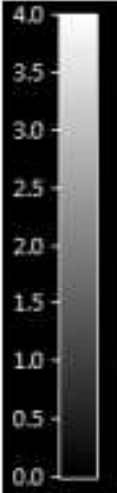

d)

a)
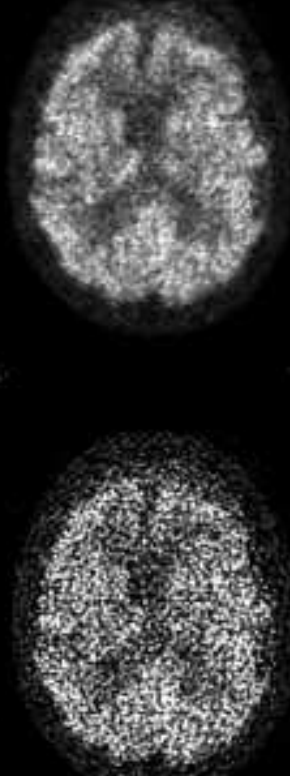

b)

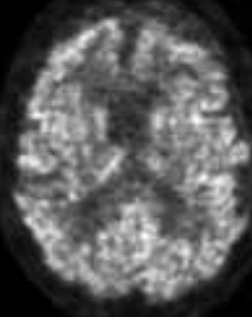

e)

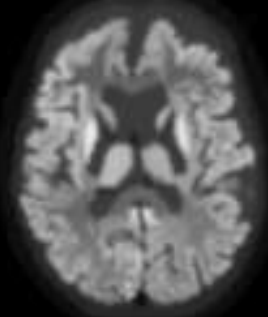

c)

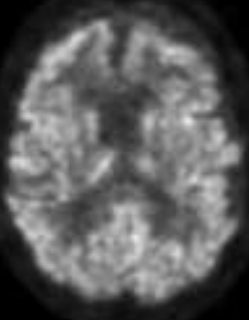

f)

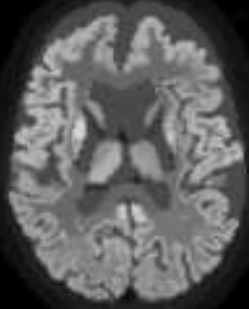


Figure 4

FDG h00015 image reconstruction (in AU) of the a) normal reconstruction, b) resolution modelling, c) Lucy-Richardson within reconstruction, d) Lucy-Richardson post reconstruction, e) Rousset within reconstruction, f) Rousset post reconstruction. Images were reconstructed using 3D OP-OSEM for 1 subset and 192 iterations. Image dimensions $256 \times 256 \times 207$, voxel size $=1.21875 \mathrm{~mm} 3$
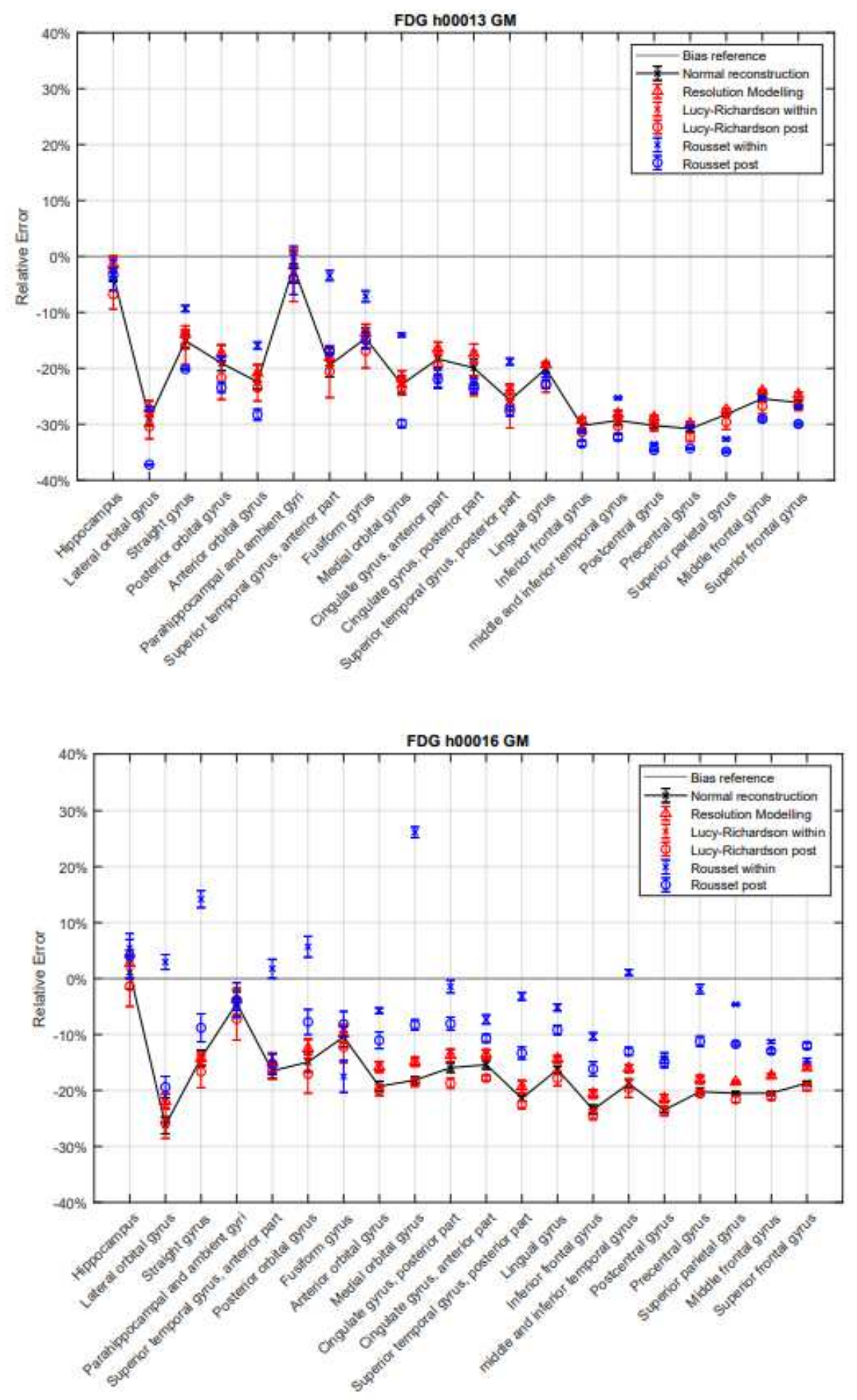

Figure 5 
Mean and standard deviations of the relative error across realisations of the radioactivity concentration in GM cortical regions ordered from small to large in volume from left to right for FDG. Two data sets (h00013 and h00016) of the FDG are shown for all 6 image options
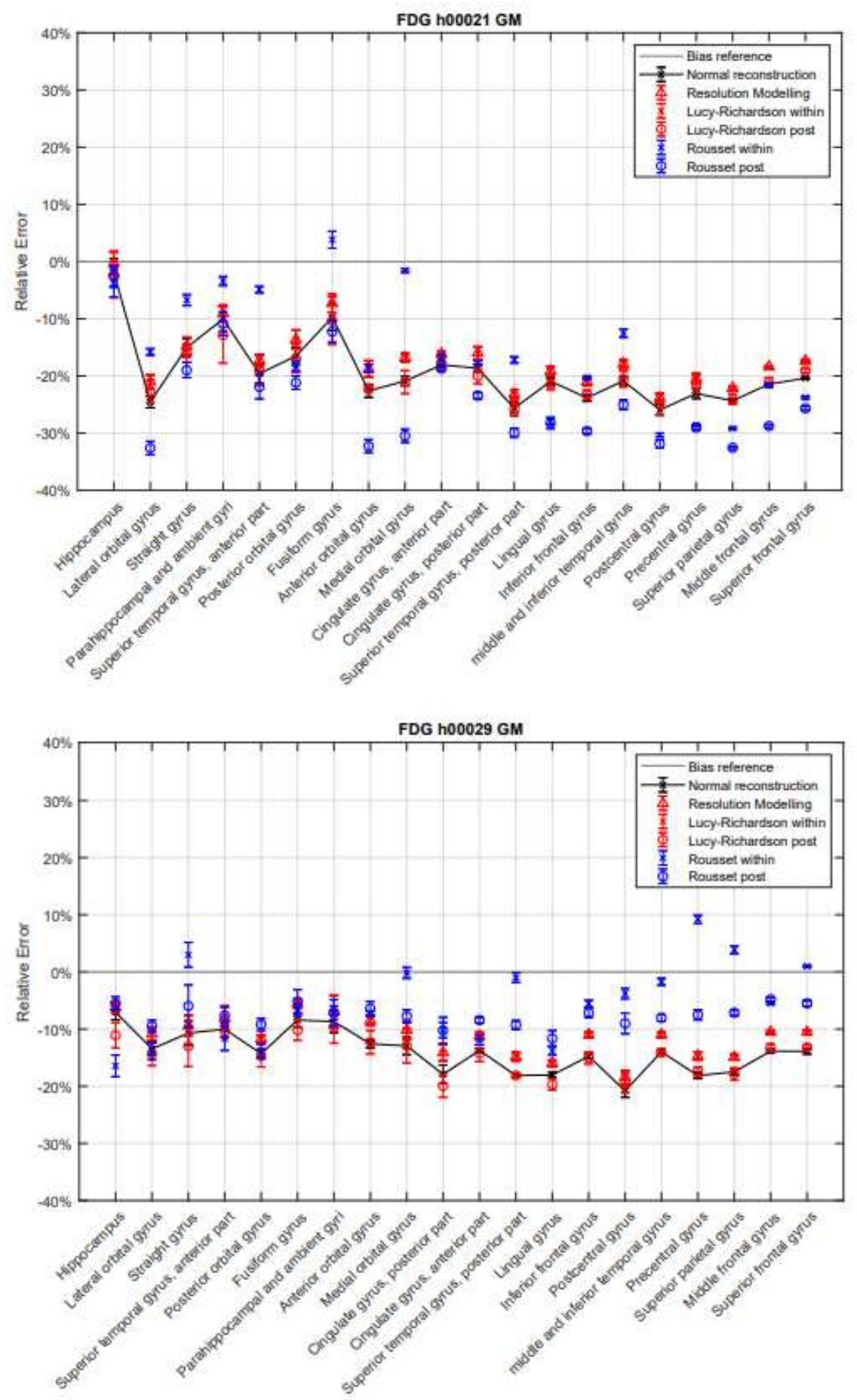

Figure 6

Mean and standard deviations of the relative error across realisations of the radioactivity concentration in GM cortical regions ordered from small to large in volume from left to right for FDG. Two data sets 
(h00021 and h00029) of the FDG are shown for all 6 image options

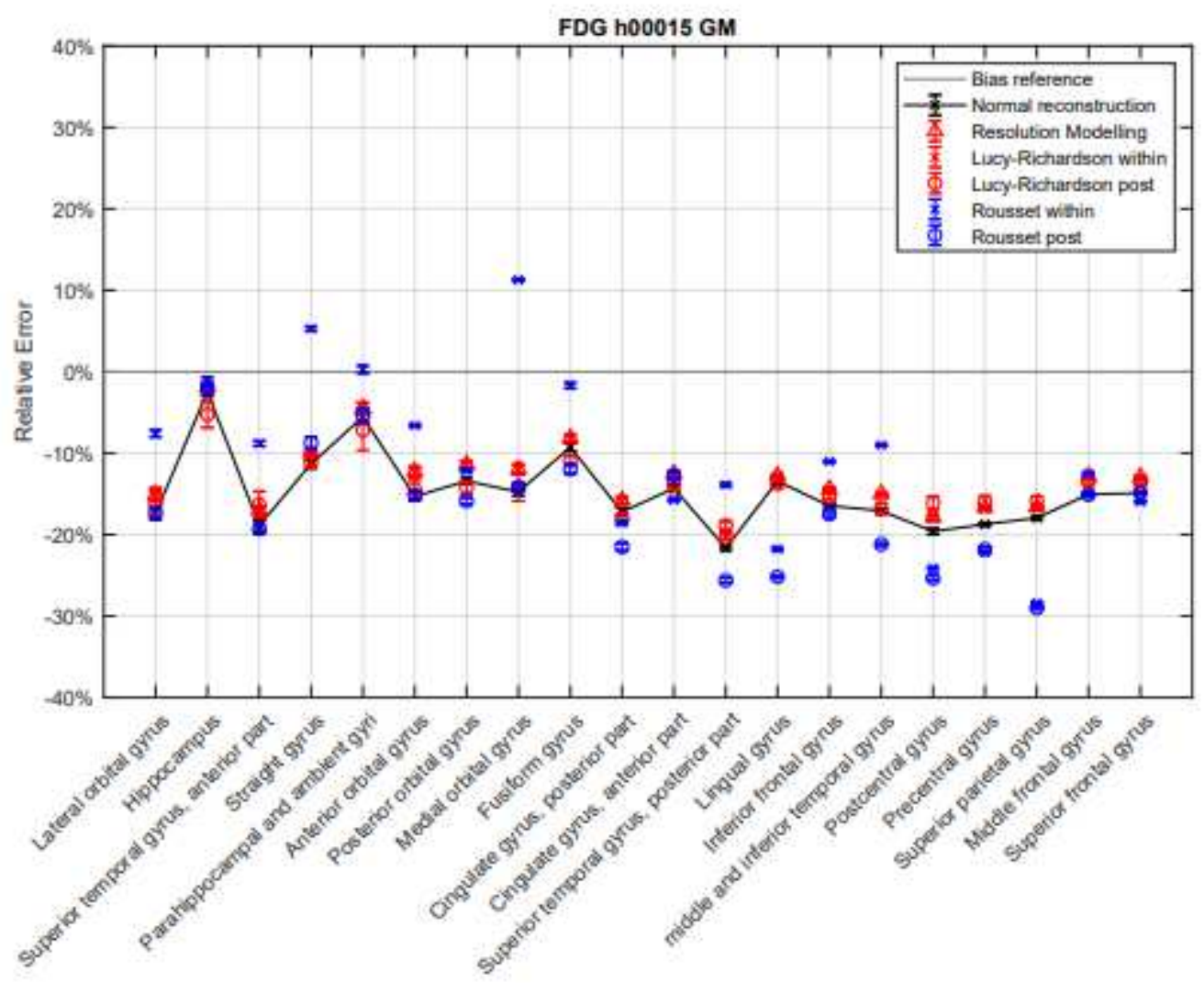

Figure 7

Mean and standard deviations of the relative error across realisations of the radioactivity concentration in GM cortical regions ordered from small to large in volume from left to right for FDG. Data from h00015 FDG are shown for all 6 image options 


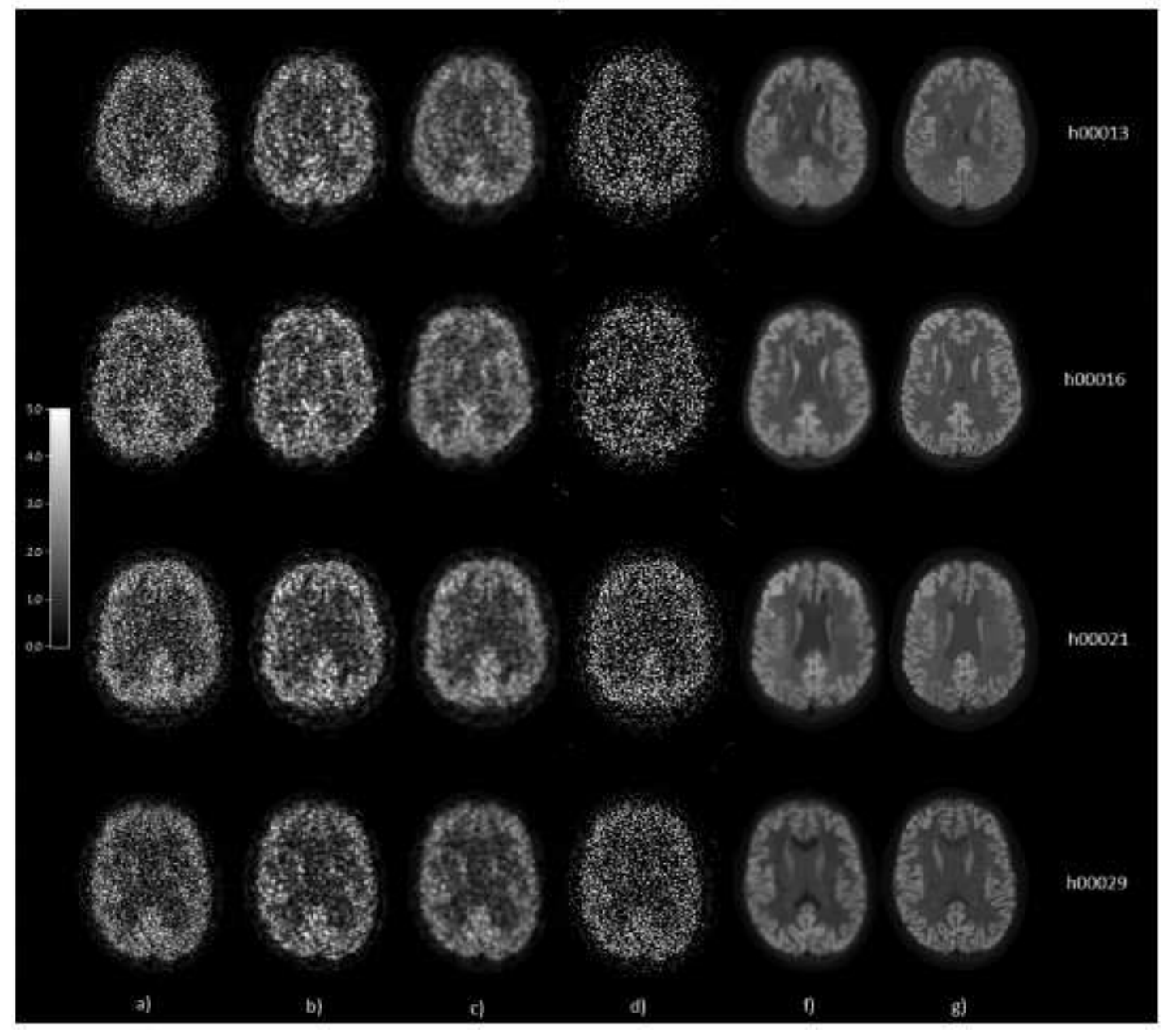

\section{Figure 8}

Remaining FDG reconstructed images (h00013, h00016, h00021 and h00029 in AU) of the a) normal reconstruction, b) resolution modelling, c) Lucy-Richardson within reconstruction, d) Lucy-Richardson post reconstruction, e) Rousset within reconstruction, f) Rousset post reconstruction. Images were reconstructed using 3D OP-OSEM for 1 subset and 192 iterations. Image dimensions $256 \times 256 \times 207$, voxel size $=1.21875 \mathrm{~mm} 3$ 

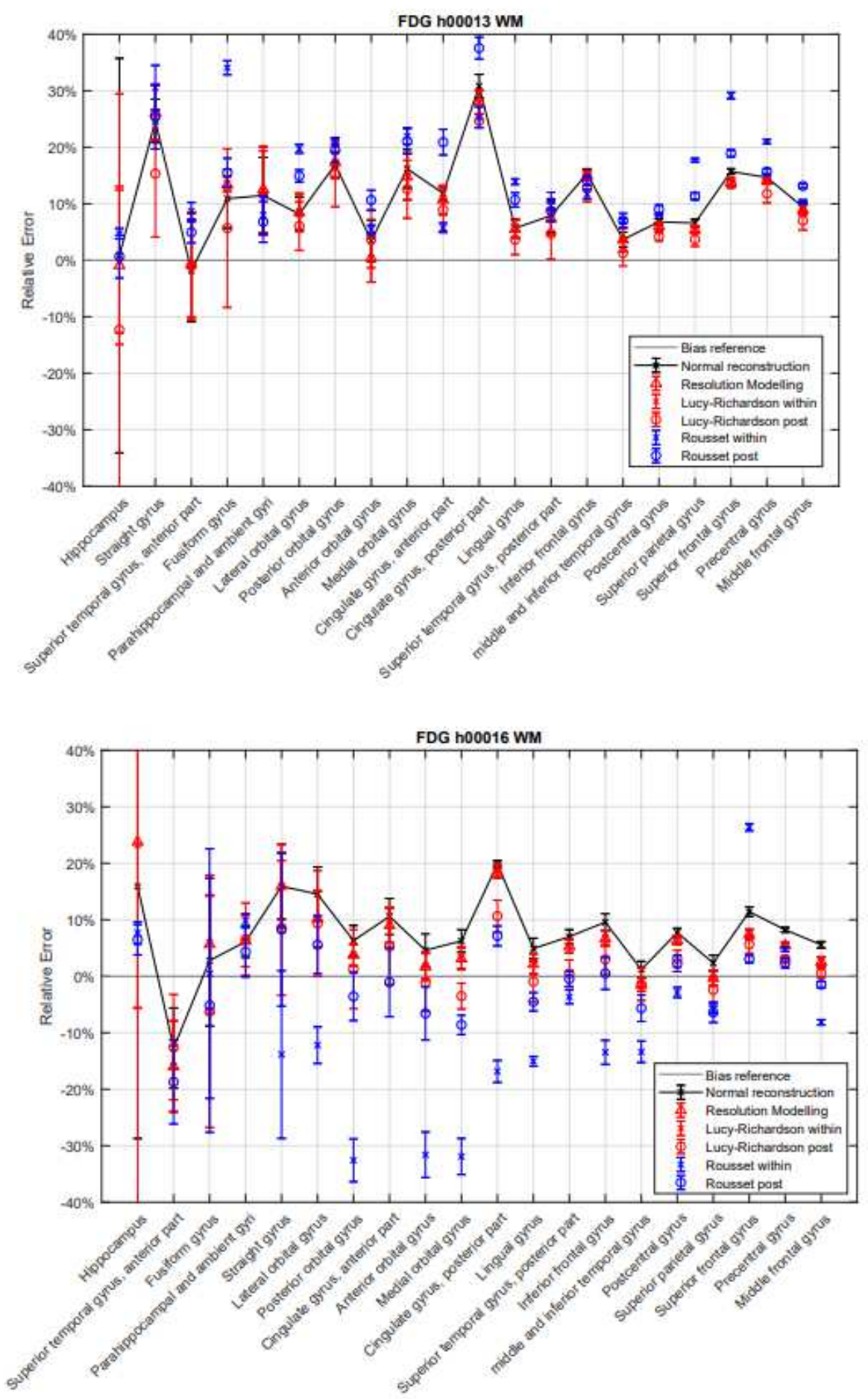

\section{Figure 9}

Mean and standard deviations of the relative error across realisations of the radioactivity concentration in WM cortical regions ordered from small to large in volume from left to right for FDG. Two data sets (h00013 and h00016) of the FDG are shown for all 6 image options 

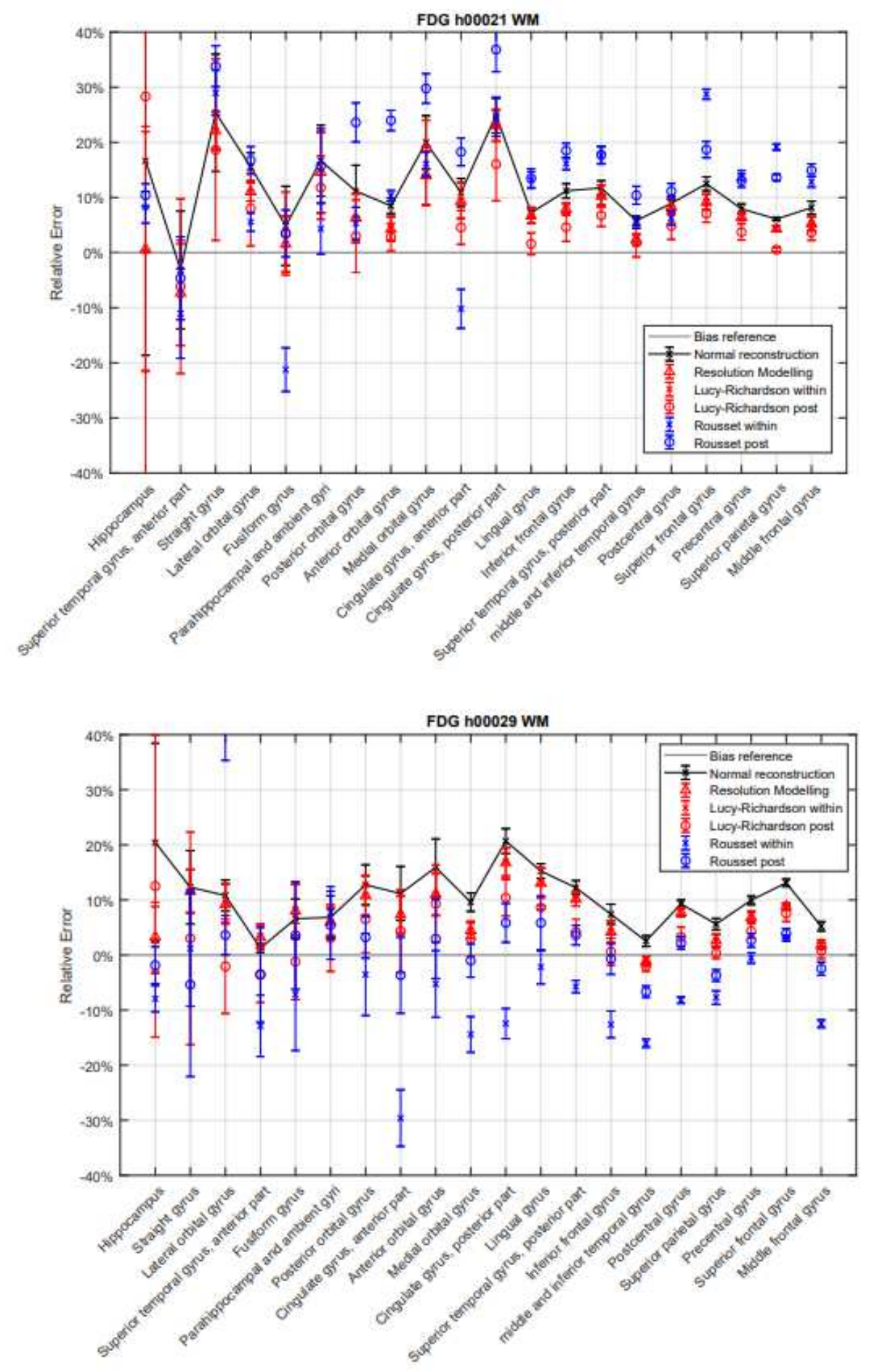

\section{Figure 10}

Mean and standard deviations of the relative error across realisations of the radioactivity concentration in WM cortical regions ordered from small to large in volume from left to right for FDG. Two data sets (h00021 and h00029) of the FDG are shown for all 6 image options 


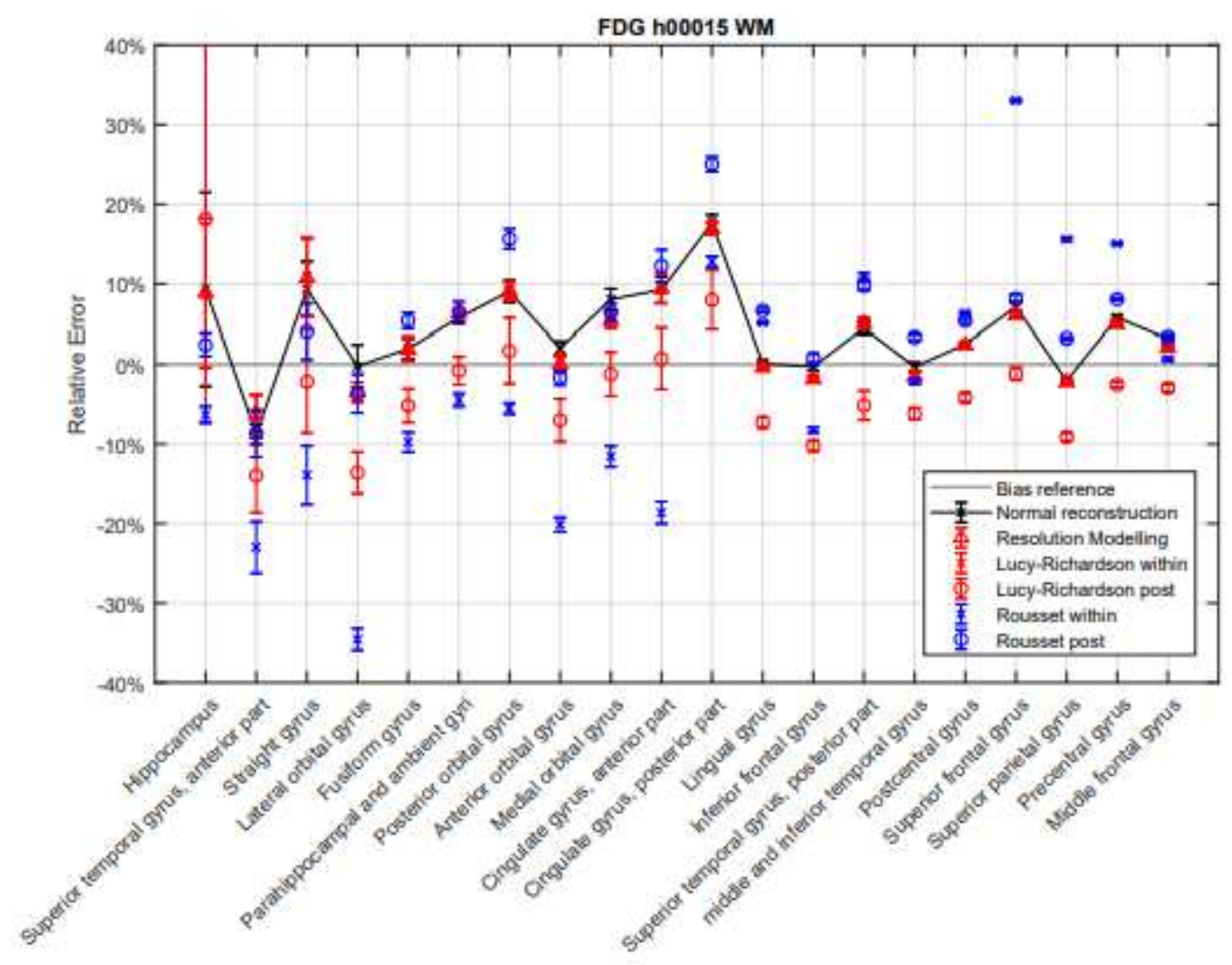

Figure 11

Mean and standard deviations of the relative error across realisations of the radioactivity concentration in WM cortical regions ordered from small to large in volume from left to right for FDG. Data from h00015 FDG are shown for all 6 image options 